\title{
NONCOMPLETE LINEAR SYSTEMS ON ABELIAN VARIETIES
}

\author{
CHRISTINA BIRKENHAKE
}

\begin{abstract}
Let $X$ be a smooth projective variety. Every embedding $X \hookrightarrow \mathbb{P}_{N}$ is the linear projection of an embedding defined by a complete linear system. In this paper the geometry of such not necessarily complete embeddings is investigated in the special case of abelian varieites. To be more precise, the properties $N_{p}$ of complete embeddings are extended to arbitrary embeddings, and criteria for these properties to be satisfied are elaborated. These results are applied to abelian varieties. The main result is: Let $(X, L)$ be a general polarized abelian variety of type $\left(d_{1}, \ldots, d_{g}\right)$ and $p \geq 1, n \geq 2 p+2$ such that $n d_{g} \geq 6$ is even, and $c \leq n^{g-1}$. The general subvector space $V \subseteq H^{0}\left(L^{n}\right)$ of codimension c satisfies the property $N_{p}$.
\end{abstract}

Let $X$ be a nonsingular projective variety defined over an algebraically closed field $K$. Every projective embedding $X \hookrightarrow \mathbb{P}_{N}$ is of the following form: There is a very ample line bundle $L$ on $X$. Its associated complete linear system $|L|$ defines the complete embedding

$$
\varphi_{L}: X \hookrightarrow \mathbb{P}\left(H^{0}(L)^{*}\right), \quad x \mapsto\left\{\sigma \in H^{0}(L) \mid \sigma(x)=0\right\} .
$$

An arbitrary embedding $\varphi: X \hookrightarrow \mathbb{P}_{N}$ is a linear projection of a complete embedding. In other words $\varphi$ factorizes as follows:

$$
\begin{array}{ccc}
X \underset{\varphi_{L}}{\hookrightarrow} & \mathbb{P}\left(H^{0}(L)^{*}\right) \\
\varphi \searrow & \begin{array}{l}
\downarrow p \\
\mathbb{P}_{N}
\end{array}
\end{array}
$$

(with a linear projection $p$ ). $\varphi$ defines a subvector space $V \subseteq H^{0}(L)$ such that $\varphi$ is the embedding induced by the linear system $|V| \subseteq|L|$ :

$$
\varphi=\varphi_{V}: X \hookrightarrow \mathbb{P}_{N}=\mathbb{P}\left(V^{*}\right), \quad x \mapsto\{\sigma \in V \mid \sigma(x)=0\} .
$$

If $V$ is a proper subvector space of $H^{0}(L)$, the morphism $\varphi_{V}$ is called a noncomplete embedding and $|V|$ is a noncomplete linear system. Given an embedding $\varphi: X \hookrightarrow$ $\mathbb{P}_{N}$, the main problems are:

1) Find equations for $X$ in $\mathbb{P}_{N}$. A strategy for solving this problem could be:

a) determine the smallest integer $k$ such that the homogeneous ideal $I_{X / \mathbb{P}_{N}}=$ $\bigoplus_{\nu>2} I_{\nu}$ of $X$ in $\mathbb{P}_{N}$ is generated by forms of degree $\leq k$,

b) determine bases for $I_{2}, \ldots, I_{k}$.

Received by the editors June 9, 1995.

1991 Mathematics Subject Classification. Primary 14C20, 14 K05.

Supported by EC Contract No. CHRXCT 940557. 
2) Describe the syzygies of $X$ in $\mathbb{P}_{N}$ : Let $S$ be the homogeneous coordinate ring of $\mathbb{P}_{N}$ and

$$
0 \rightarrow \bigoplus_{j} S(-j)^{b_{r, j}} \stackrel{\varphi_{r-1}}{\longrightarrow} \ldots \stackrel{\varphi_{0}}{\longrightarrow} \bigoplus_{j} S(-j)^{b_{0, j}} \rightarrow M \rightarrow 0
$$

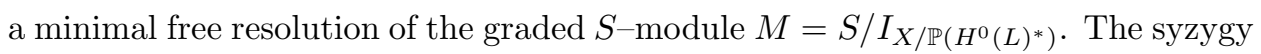
modules $\operatorname{im} \varphi_{i}$ reflect subtle information about the intrinsic geometry of $X$. A first step in determining the syzygy modules is to compute the graded Betti numbers $b_{i j}$.

Green, Lazarsfeld, Ein and many others studied these problems using Koszul cohomology. However, their methods required the embedding in question to be projectively normal, and projectively normal embeddings are necessarily complete. In order to adjust the Koszul cohomology methods to noncomplete embeddings $\varphi_{V}: X \hookrightarrow \mathbb{P}_{N}$ the first step is to modify the notion of projective normality: the subvector space $V \subseteq H^{0}(L)$ is called $k$-normal, if the canonical map

$$
S^{k} V \rightarrow H^{0}\left(L^{k}\right)
$$

is surjective. In these terms $\varphi_{V}: X \hookrightarrow \mathbb{P}_{N}$ is projectively normal if and only if $V$ is $k$-normal for $k \geq 1$. In particular, $V=H^{0}(L)$. For the investigation of noncomplete linear systems $|V| \subseteq|L|$ it turns out to be convenient to replace the condition of projective normality by $k$-normality for $k \geq 2$. Furthermore we replace the $S$-module $M$ in 2) by the graded $S$-module

$$
R=\bigoplus_{\nu \geq 0} R_{\nu} \quad \text { with } \quad R_{\nu}:= \begin{cases}K & \text { if } \quad \nu=0 \\ V & \text { if } \nu=1 \\ H^{0}\left(L^{\nu}\right) & \text { if } \quad \nu \geq 2 .\end{cases}
$$

Notice that for projectively normal embeddings $R=M$. The next step is to modify the properties $N_{p}$ introduced by M. Green: Let

$$
0 \rightarrow \bigoplus_{j} S(-j)^{\tilde{b}_{r, j}} \rightarrow \ldots \rightarrow \bigoplus_{j} S(-j)^{\tilde{b}_{0, j}} \rightarrow R \rightarrow 0
$$

be a minimal free resolution. For an integer $p \geq 0$ the subvector space $V$ is said to satisfy the property $\widetilde{N}_{p}$, if

$$
\tilde{b}_{0, j}=\left\{\begin{array}{ll}
1 & \text { if } j=0, \\
0 & \text { otherwise, }
\end{array} \quad \text { and } \quad \tilde{b}_{i j}=0 \text { for } 1 \leq i \leq p \text { and } j>i+1 .\right.
$$

In these terms $\widetilde{N}_{0}$ is equivalent to $V$ being $k$-normal for $k \geq 2, \widetilde{N}_{1}$ means that the ideal $I_{X / \mathbb{P}_{N}}$ of $X$ in $\mathbb{P}\left(V^{*}\right)=\mathbb{P}_{N}$ is generated by quadrics, $\widetilde{N}_{2}$ means that the syzygies of the quadric generators of $I_{X / \mathbb{P}_{N}}$ admit only linear polynomials as coefficients, etc. Notice also that in the case of a complete linear system $|V|=|L|$ the properties $\widetilde{N}_{p}$ and $N_{p}$ coincide. If we use this setup similarly as in the case of complete embeddings, Koszul cohomology methods apply. However, there is one obstruction. There is no cohomological criterion for 2-normality.

The 2-normality of a linear system has to be checked separately. The first observation is that one can only expect to prove 2-normality for a general subvector space $V \subseteq H^{0}(L)$, since certainly there exist linear projections $p: \mathbb{P}\left(H^{0}(L)^{*}\right)--\longrightarrow$ $\mathbb{P}\left(V^{*}\right)$ leading for example to singularities of the image of $X$ in $\mathbb{P}\left(V^{*}\right)$. On the other hand there seems to be no general criterium for $2-$ normality. The methods for proving 2-normality depend intrinsically on the variety $X$ as well as the linear system $L$. So far the author has studied $2-$ normality and the property $\widetilde{N}_{p}$ of linear 
systems on curves, projective spaces and abelian varieties. In this paper the results corresponding to abelian varieties are presented.

The paper is organized as follows: In Sections 1 and 2 Koszul cohomology methods are applied to prove a cohomological criterion for the property $\widetilde{N}_{p}$ for noncomplete linear systems. In Section 3 we study the homogeneous ideal $I_{X / \mathbb{P}_{N}}$. The second part of the paper, Sections 4 to 7 , deals with noncomplete linear systems on complex abelian varieties. Let $(X, L)$ be a general polarized abelian variety. In Section 4 we apply the Theta Multiplication Formula to study 2-normality of subvector spaces $V \subseteq H^{0}(L)$. According to a theorem of Kempf (see [K]) the complete linear system $\left|L^{n}\right|$ satisfies property $N_{p}, p \geq 1$, if $n \geq 2 p+2$. The main theorem of Section 6 is an analogon of Kempf's theorem for noncomplete linear systems (see Corollary 6.2). In Section 7 again we use the Theta Multiplication Formula to determine explicit equations for abelian varieties in $\mathbb{P}_{N}$.

\section{1. $k$-NORMALITY}

Let $X$ be a closed subvariety of the projective space $\mathbb{P}_{N}$ defined over an algebraically closed field $K$. For projectively normal embeddings $X \hookrightarrow \mathbb{P}_{N}$ there are methods to study the homogeneous ideal of $X$ in $\mathbb{P}_{N}$ (see for example [G1], [G2], [L1]). But projectively normal subvarieties of $\mathbb{P}_{N}$ are necessarily completely embedded. In order to study noncompletely embedded varieties it turns out to be useful to extend slightly the notion of projective normality. For this we start studying $k$-normal linear systems.

Let $L$ be an ample line bundle on the projective variety $X$. A subvector space $V \subseteq H^{0}(L)$ as well as its associated sublinear system $|V| \subseteq|L|$ is called base point free (respectively (very) ample), if $|V|$ is base point free (respectively (very) ample). For a base point free subvector space $V \subseteq H^{0}(L)$ denote by $\varphi_{V}$ the morphism defined by $|V|$ :

$$
\varphi_{V}: X \longrightarrow \mathbb{P}_{N}=\mathbb{P}\left(V^{*}\right), x \mapsto\{\sigma \in V \mid \sigma(x)=0\},
$$

where $\mathbb{P}\left(V^{*}\right)$ is the projective space of hyperplanes in $V$. Let $k$ be an integer $\geq 1$ and denote by $S^{k} V$ the $k$-th symmetric product of $V$. Recall that $V$ is $k$-normal, if the canonical map $S^{k} V \longrightarrow H^{0}\left(L^{k}\right)$ is surjective. Via the canonical identifications $S^{k} V=S^{k} H^{0}\left(\mathcal{O}_{\mathbb{P}_{N}}(1)\right)=H^{0}\left(\mathcal{O}_{\mathbb{P}_{N}}(k)\right), k$-normality of $V$ is equivalent to surjectivity of the restriction map $H^{0}\left(\mathcal{O}_{\mathbb{P}_{N}}(k)\right) \longrightarrow H^{0}\left(X, L^{k}\right)$, i.e. the hypersurfaces of degree $k$ cut out the complete linear system $\left|L^{k}\right|$ on $X$. In particular, a normal subvariety $X$ of $\mathbb{P}_{N}$ is projectively normal if and only if it is $k$-normal for $k \geq 1$.

(1.1) Proposition. Let $V \subseteq H^{0}(L)$ be a subvector space. Suppose for some $k \geq 1$ $V$ is $k$-normal and $L^{k}$ is very ample. Then $V$ is very ample as well.

Proof. The hypothesis implies that $V$ is base point free, so $\varphi_{V}: X \longrightarrow \mathbb{P}\left(V^{*}\right)$ is a morphism. Denote by $\varphi_{L^{k}}: X \hookrightarrow \mathbb{P}\left(H^{0}\left(L^{k}\right)^{*}\right)$ the embedding defined by $\left|L^{k}\right|$, and by $\sigma: \mathbb{P}\left(V^{*}\right) \hookrightarrow \mathbb{P}\left(S^{k} V^{*}\right)$ the Veronese embedding. By assumption $\psi$ : $\mathbb{P}\left(H^{0}\left(L^{k}\right)^{*}\right) \hookrightarrow \mathbb{P}\left(S^{k} V^{*}\right)$ is an embedding. Then the following diagram commutes:

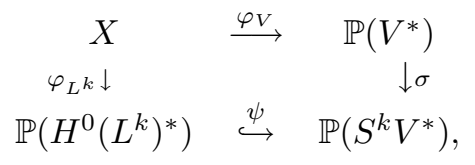

and thus $\varphi_{V}$ is an embedding. 
(1.2) Remark. The same proof shows also:

If $V \subseteq H^{0}(L)$ is $k$-normal and $L^{k}$ is base point free for some $k \geq 1$, then $V$ is base point free.

Consider the trivial vector bundle $V \otimes_{K} \mathcal{O}_{X}$ on $X . V$ being base point free, the evaluation map $V \otimes_{K} \mathcal{O}_{X} \longrightarrow L$ is surjective. Hence its kernel $M_{V}$ is a vector bundle of rank $\operatorname{dim} V-1$, and there is a short exact sequence of vector bundles on $X$

$$
0 \longrightarrow M_{V} \longrightarrow V \otimes_{K} \mathcal{O}_{X} \longrightarrow L \longrightarrow 0
$$

The vector bundle $M_{V}$ enables one to check $k$-normality in terms of vanishings of certain cohomology groups.

(1.3) Lemma. Suppose $V \subseteq H^{0}(L)$ is a 2-normal subvector space and $k_{0}>2$ an integer.

a) If $H^{1}\left(M_{V} \otimes L^{k}\right)=0$ for $2 \leq k \leq k_{0}-1$, then $V$ is $k$-normal for $2 \leq k \leq k_{0}$.

b) Suppose $H^{1}\left(L^{i}\right)=0$ for $2 \leq i \leq k_{0}-1$. Then the following two conditions are equivalent:

i) $V$ is $k$-normal for $2 \leq k \leq k_{0}$,

ii) $H^{1}\left(M_{V} \otimes L^{k}\right)=0$ for $2 \leq k \leq k_{0}-1$.

Proof. It suffices to consider the canonical maps $V^{\otimes k} \longrightarrow H^{0}\left(L^{k}\right)$ instead of $S^{k} V \rightarrow$ $H^{0}\left(L^{k}\right)$, the natural projections $V^{\otimes k} \longrightarrow S^{k} V$ being surjective for $k \geq 1$. Consider the following factorization of $V^{\otimes k} \rightarrow H^{0}\left(L^{k}\right)$ :

$$
\begin{aligned}
V^{\otimes k} & \stackrel{\mu_{2}}{\longrightarrow} V^{\otimes(k-2)} \otimes H^{0}\left(L^{2}\right) \rightarrow V^{\otimes(k-3)} \otimes H^{0}\left(L^{3}\right) \\
& \rightarrow \ldots \rightarrow V \otimes H^{0}\left(L^{k-1}\right) \rightarrow H^{0}\left(L^{k}\right)
\end{aligned}
$$

Here $\mu_{2}$ is surjective, $V$ being 2 -normal. As for a) and b) ii) $\Rightarrow$ i): It remains to show that $V \otimes H^{0}\left(L^{i}\right) \rightarrow H^{0}\left(L^{i+1}\right)$ is surjective for $2 \leq i \leq k_{0}-1$. Consider the long exact cohomology sequence associated to (1) tensored with $L^{i}$ :

$$
\longrightarrow V \otimes H^{0}\left(L^{i}\right) \rightarrow H^{0}\left(L^{i+1}\right) \rightarrow H^{1}\left(M_{V} \otimes L^{i}\right) \rightarrow V \otimes H^{1}\left(L^{i}\right) \longrightarrow .
$$

Hence $H^{1}\left(M_{V} \otimes L^{i}\right)=0$ implies the surjectivity of $V \otimes H^{0}\left(L^{i}\right) \longrightarrow H^{0}\left(L^{i+1}\right)$. As for b) i) $\Rightarrow$ ii): It suffices to notice that, since $H^{1}\left(L^{i}\right)=0$ for $2 \leq i \leq k_{0}-1$, $H^{1}\left(M_{V} \otimes L^{i}\right)=0$ if and only if $V \otimes H^{0}\left(L^{i}\right) \longrightarrow H^{0}\left(L^{i+1}\right)$ is surjective.

Under weak hypotheses base point free, 2-normal subvector spaces $V \subseteq H^{0}(L)$ are always $k$-normal for $k>\operatorname{dim} X$. This is a consequence of the generalized Castelnuovo Lemma for noncomplete linear systems.

(1.4) Proposition (Generalized Castelnuovo Lemma). Suppose $\mathcal{F}$ is a coherent sheaf on $X$ satisfying $H^{i}\left(\mathcal{F} \otimes L^{-i}\right)=0$ for $i \geq 1$. Then for every base point free subvector space $V \subseteq H^{0}(L)$

$$
H^{1}\left(M_{V} \otimes \mathcal{F} \otimes L^{i}\right)=0 \quad \text { for } \quad i \geq 0
$$

See $[\mathrm{M}]$, Theorem 2, for the version for complete linear systems, i.e. $V=H^{0}(L)$. The proof is a slight modification of the proof in $[\mathrm{M}]$ : it suffices to choose the section $s \in H^{0}(L)$ within $V$.

Applying Proposition 1.4 to the sheaf $\mathcal{F}=L^{\operatorname{dim} X+1}$ gives 
(1.5) Corollary. Suppose $V \subseteq H^{0}(L)$ is base point free and $H^{i}\left(L^{j}\right)=0$ for $i, j \geq 1$. Then

$$
H^{1}\left(M_{V} \otimes L^{k}\right)=0 \quad \text { for } \quad k>\operatorname{dim} X .
$$

An immediate consequence is

(1.6) Corollary. Suppose $L$ is a nonspecial line bundle on a curve $C$. Every base point free, 2-normal subvector space $V \subseteq H^{0}(L)$ is $k$-normal for $k \geq 2$.

\section{The Property $\widetilde{N}_{p}$}

Mark Green introduced in [G1] the property $N_{p}$ for complete linear systems. The aim of this section is to generalize this property to arbitrary (not necessarily complete) base point free linear systems. The extended version of the property $N_{p}$ will be denoted by $\widetilde{N}_{p}$. The main result of this section is Theorem 2.4, a sufficient criterion for the property $\widetilde{N}_{p}$.

Suppose $L$ is an ample line bundle on a projective variety $X$ of dimension $n$, and $V \subseteq H^{0}(L)$ a base point free subvector space with associated morphism $\varphi_{V}$ : $X \longrightarrow \mathbb{P}_{N}$. The symmetric algebra $S=\bigoplus_{p \geq 0} S^{p} V$ is the homogeneous coordinate ring of $\mathbb{P}_{N}$. Define the graded $S$-module

$$
R:=\bigoplus_{p \geq 0} R_{p}, \quad \text { where } \quad R_{p}=\left\{\begin{array}{lll}
K, & \text { if } & p=0, \\
V & \text { if } & p=1, \\
H^{0}\left(L^{p}\right) & \text { if } & p \geq 2 .
\end{array}\right.
$$

Denote by $I=I_{X / \mathbb{P}_{N}}=\bigoplus_{p \geq 2} I_{p}$ the homogeneous ideal of $X$ in $\mathbb{P}_{N}$, i.e. $I_{p}=$ $\operatorname{ker}\left(S^{p} V \rightarrow R_{p}\right)$. Choose a minimal free resolution of $R$

$$
0 \rightarrow \bigoplus_{j} S(-j)^{\tilde{b}_{r j}} \rightarrow \ldots \rightarrow \bigoplus_{j} S(-j)^{\tilde{b}_{1 j}} \rightarrow \bigoplus_{j} S(-j)^{\tilde{b}_{0 j}} \rightarrow R \rightarrow 0 .
$$

The integers $\tilde{b}_{i j}$, the so-called graded Betti numbers, are uniquely determined by $R$.

(2.1) Definition. The subvector space $V$ is said to satisfy the property $\widetilde{N}_{0}$, if

$$
\tilde{b}_{0 j}= \begin{cases}1 & \text { if } j=0 \\ 0 & \text { otherwise. }\end{cases}
$$

For $p \geq 1$ we say that $V$ satisfies the property $\widetilde{N}_{p}$, if $\widetilde{N}_{p-1}$ holds and $\tilde{b}_{p j}=0$ for $j>p+1$.

In other words, $V$ satisfies the property $\widetilde{N}_{p}$, if $R$ admits a minimal free resolution of the form

$$
\begin{aligned}
\rightarrow \bigoplus_{j \geq p+2} S(-j)^{\tilde{b}_{p+1, j}} & \rightarrow S(-p-1)^{\tilde{b}_{p, p+1}} \rightarrow \ldots \\
\ldots & \rightarrow S(-3)^{\tilde{b}_{23}} \rightarrow S(-2)^{\tilde{b}_{12}} \rightarrow S \rightarrow R \rightarrow 0 .
\end{aligned}
$$

Thus for $p \geq 1$ the properties $\widetilde{N}_{p}$ lead to the same geometric interpretation as the properties $N_{p}$ in the complete case:

$$
\begin{aligned}
& \widetilde{N}_{0} \Longleftrightarrow V \text { is } k \text {-normal for } k \geq 2, \\
& \widetilde{N}_{1} \Longleftrightarrow \widetilde{N}_{0} \text { holds and the ideal } I_{X / \mathbb{P}_{N}} \text { of } X \text { in } \mathbb{P}_{N} \text { is generated by quadrics, }
\end{aligned}
$$




$$
\begin{gathered}
\widetilde{N}_{2} \Longleftrightarrow \widetilde{N}_{1} \text { holds and the module of syzygies among the quadratic } \\
\text { generators } Q_{i} \text { of } I_{2} \text { is generated by linear relations } \\
\sum_{i} \ell_{i} Q_{i}=0 \text { with linear polynomials } \ell_{i} \text {, etc. }
\end{gathered}
$$

The property $\widetilde{N}_{p}$ can be characterized by the vanishing of certain Koszul cohomology groups. For this consider the Koszul complex associated with $V$ :

$$
\begin{aligned}
& K \bullet=K(V)_{\bullet}: \\
& \ldots \rightarrow \bigwedge^{p+1} V \otimes_{K} S(-p-1) \rightarrow \bigwedge^{p} V \otimes_{K} S(-p) \rightarrow \bigwedge^{p-1} V \otimes_{K} S(-p+1) \rightarrow \ldots
\end{aligned}
$$

and the homogeneous degree $(p+q)$-part of the twisted complex

$$
(K \bullet \otimes R)_{p+q}: \ldots \rightarrow \bigwedge^{p+1} V \otimes_{K} R_{q-1} \stackrel{d_{p+1, q-1}}{\longrightarrow} \bigwedge^{p} V \otimes R_{q} \stackrel{d_{p, q}}{\longrightarrow} \bigwedge^{p-1} V \otimes R_{q+1} \rightarrow \ldots
$$

Define the Koszul cohomology group $\mathcal{K}_{p, q}(V, R)$ to be the $p$-th cohomology group of the complex $\left(K_{\bullet} \otimes R\right)_{p+q}$ :

$$
\mathcal{K}_{p, q}(V, R):=\frac{\operatorname{ker} d_{p, q}}{\operatorname{im} d_{p+1, q-1}} .
$$

(2.2) Proposition. For $p \geq 0$ the following two conditions are equivalent:

i) $V$ satisfies the property $\widetilde{N}_{p}$,

ii) $\mathcal{K}_{i, q}(V, R)=0$ for $0 \leq i \leq p$ and $q \geq 2$.

The proof is an immediate modification of the corresponding proof for complete linear systems (see [G2]). If the codimension of $X$ in $\mathbb{P}_{N}$ is sufficiently large, one can improve the above criterion. In fact it suffices to check the vanishing of the "highest" Koszul cohomology group (see also [G-L] for the corresponding statement for complete linear systems).

(2.3) Proposition. For $0 \leq p<\operatorname{codim}\left(X, \mathbb{P}\left(V^{*}\right)\right)$ the following conditions are equivalent:

i) $V$ satisfies the property $\widetilde{N}_{p}$,

ii) $\mathcal{K}_{p, q}(V, R)=0$ for $q \geq 2$.

Proof. It suffices to show: If $k \leq p$ and $\mathcal{K}_{k, q}(V, R)=0$ for all $q \geq 2$, then $\mathcal{K}_{k-1, q}(V, R)=0$ for all $q \geq 2$. Taking into account that $\operatorname{dim} \mathcal{K}_{i, j}(V, R)=\tilde{b}_{i, j+i}$ (see [G1], Theorem 1.b.4), equivalently it suffices to show:

$$
\text { if } k \leq p \text { and } \tilde{b}_{k, j}=0 \text { for all } j \geq k+2 \text {, then } \tilde{b}_{k-1, j}=0 \text { for all } j \geq k+1 \text {. }
$$

First we show that $\operatorname{Ext}_{S}^{k}(R, S)=0$ for $k<\operatorname{codim}\left(X, \mathbb{P}\left(V^{*}\right)\right)$.

For this recall that by definition $\operatorname{Ext}_{S}^{k}(R, S)=0$ for $k<\operatorname{depth}(\operatorname{ann}(R))$, where $\operatorname{ann}(R)$ denotes the annihilator of $R$ in $S$. Certainly $\operatorname{ann}(R)=I_{X / \mathbb{P}_{N}}$, and thus $\operatorname{depth} I_{X / \mathbb{P}_{N}}=\operatorname{codim} I(V)=\operatorname{codim}\left(X, \mathbb{P}\left(V^{*}\right)\right)$ (see [Ma]). This proves the claim.

It follows that dualizing the minimal free resolution (1) of $R$ yields an exact sequence

$$
0 \rightarrow R^{*} \rightarrow \bigoplus_{j} S^{*}(j)^{\tilde{b}_{0 j}} \rightarrow \ldots \rightarrow \bigoplus_{j} S^{*}(j)^{\tilde{b}_{k-1, j}} \stackrel{\delta}{\rightarrow} \bigoplus_{j} S^{*}(j)^{\tilde{b}_{k, j}} \rightarrow T \rightarrow 0
$$

for $k \leq p<\operatorname{codim}\left(X, \mathbb{P}\left(V^{*}\right)\right)$ with $T=\operatorname{coker} \delta$. Obviously this is a minimal free resolution of $T$, implying

$$
\max \left\{j \mid \tilde{b}_{k-1, j} \neq 0\right\}<\max \left\{j \mid \tilde{b}_{k, j} \neq 0\right\} .
$$


By assumption the right-hand side of this inequality is $\leq k+1$, hence $\tilde{b}_{k-1, j}=0$ for $j \geq k+1$, which proves $(*)$.

The next aim is to describe the properties $\widetilde{N}_{p}$ cohomogically. For this recall the vector bundle $M_{V}$ defined in Section 1.

(2.4) Theorem. Suppose $V \subseteq H^{0}(L)$ is a 2-normal subvector space and $p$ is an integer $\geq 0$. Then $\widetilde{N}_{p}$.

a) If $H^{1}\left(\bigwedge^{i} M_{V} \otimes L^{k}\right)=0$ for $1 \leq i \leq p+1$ and $k \geq 1$, then $V$ satisfies property

b) If $p<\operatorname{codim}\left(X, \mathbb{P}\left(V^{*}\right)\right)$ and $H^{1}\left(\bigwedge^{p+1} M_{V} \otimes L^{k}\right)=0$ for $k \geq 1$, then $V$ satisfies property $\widetilde{N}_{p}$.

For the proof recall the following well known lemma on exterior products of short exact sequences of vector bundles.

(2.5) Lemma. Let $0 \rightarrow E \rightarrow F \rightarrow L \rightarrow 0$ be an exact sequence of vector bundles $E$ and $F$ and a line bundle $L$ on $X$. Then for $i \geq 1$ the following sequence is exact:

$$
0 \rightarrow \bigwedge^{i} E \rightarrow \bigwedge^{i} F \rightarrow \bigwedge^{i-1} E \otimes L \rightarrow 0
$$

For a proof see [Hi], Theorem 4.1.3, p. 56 .

Proof of Theorem 2.4. Applying Lemma 2.5 to the exact sequence 1(1) defining $M_{V}$ yields

$$
0 \rightarrow \bigwedge^{i+1} M_{V} \otimes L^{k-1} \rightarrow \bigwedge^{i+1} V \otimes L^{k-1} \rightarrow \bigwedge^{i} M_{V} \otimes L^{k} \rightarrow 0 .
$$

Consider the following diagram with exact rows and columns

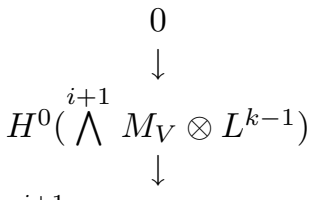

$$
\begin{aligned}
& \stackrel{i+1}{\wedge} V \otimes H^{0}\left(L^{k-1}\right) \quad \square
\end{aligned}
$$

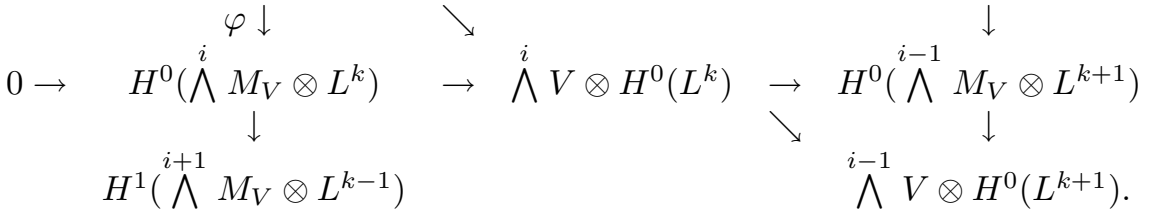

For $k \geq 3$, the diagonal is a section of the Koszul complex $\left(K_{\bullet} \otimes R\right)_{i+k}$. By assumption $\varphi$ is surjective for $0 \leq i \leq p$ and $k \geq 2$. Hence the diagonal sequence is exact in $\bigwedge^{i} V \otimes H^{0}\left(L^{k}\right)=\bigwedge^{i} V \otimes R_{k}$, i.e.

$$
\mathcal{K}_{i, k}(V, R)=0 \quad \text { for } 0 \leq i \leq p \text { and } k \geq 3 .
$$

Now suppose $k=2$. As before, the assumption implies that $\varphi$ is surjective and hence the sequence

$$
\bigwedge^{i+1} V \otimes H^{0}(L) \stackrel{\alpha}{\longrightarrow} \bigwedge^{i} V \otimes H^{0}\left(L^{2}\right) \stackrel{\beta}{\longrightarrow} \bigwedge^{i-1} V \otimes H^{0}\left(L^{3}\right)
$$


is exact. By definition of the Koszul sequence the homomorphism $\alpha$ factorizes as follows:

$$
\begin{aligned}
& \stackrel{i+1}{\bigwedge} V \otimes H^{0}(L) \quad \stackrel{\gamma \otimes i d_{H^{0}(L)}}{\longrightarrow} \quad \bigwedge^{i} V \otimes V \otimes H^{0}(L) \\
& \alpha \searrow \quad \swarrow i d \otimes \mu \\
& \bigwedge^{i} V \otimes H^{0}\left(L^{2}\right) .
\end{aligned}
$$

Here $\gamma: \bigwedge^{i+1} V \rightarrow \bigwedge^{i} V \otimes V$ is the dual to the canonical homomorphism $\bigwedge^{i} V^{*} \otimes$ $V^{*} \rightarrow \bigwedge^{i+1} V^{*}$ and $\mu: V \otimes H^{0}(L) \rightarrow H^{0}\left(L^{2}\right)$ is the multiplication map. Certainly $\operatorname{im} \mu \mid V \otimes V=\operatorname{im} \mu=H^{0}\left(L^{2}\right)$, since $V$ is $2-$ normal, hence $\operatorname{im} \alpha \mid \bigwedge^{i+1} V \otimes V=$ $\operatorname{im} \alpha=\operatorname{ker} \beta$. In particular, $\bigwedge^{i+1} V \otimes V \rightarrow \bigwedge^{i} V \otimes H^{0}\left(L^{2}\right) \rightarrow \bigwedge^{i-1} V \otimes H^{0}\left(L^{3}\right)$ is exact, implying

$$
\mathcal{K}_{i, 2}(V, R)=0 \quad \text { for } \quad 0 \leq i \leq p .
$$

Finally, Propositions 2.2 and 2.3 imply assertions a) and b) respectively.

(2.6) Corollary. Suppose $X=C$ is a curve, and $V \in H^{0}(L)$ is a 2-normal subvector space. If for some $p \geq 0$

$$
H^{1}\left(C, \bigwedge^{i} M_{V} \otimes L\right)=0 \quad \text { for } \quad 1 \leq i \leq p+1,
$$

then $V$ satisfies property $\widetilde{N}_{p}$.

Proof. Let $D \in|L|$. Restricting $\bigwedge^{i} M_{V} \otimes L^{k}$ to $D$ gives the exact cohomology sequence

$$
H^{1}\left(C, \bigwedge^{i} M_{V} \otimes L^{k-1}\right) \rightarrow H^{1}\left(C, \bigwedge^{i} M_{V} \otimes L^{k}\right) \rightarrow H^{1}\left(D, \bigwedge^{i} M_{V} \otimes L^{k} \mid D\right)=0 .
$$

So inductively the vanishing of $H^{1}\left(\bigwedge^{i} M_{V} \otimes L\right)$ yields the vanishings

$$
H^{1}\left(C, \bigwedge^{i} M_{V} \otimes L^{k}\right)=0 \quad \text { for } k \geq 2
$$

(2.7) Remark. The proof of Theorem 2.4 shows the stronger statement:

A 2-normal subvector space $V \subseteq H^{0}(X, L)$ satisfies property $\widetilde{N}_{p}$ if and only if the homomorphisms $H^{1}\left(\bigwedge^{i} M_{V} \otimes L^{k}\right) \rightarrow \bigwedge^{i} V \otimes H^{1}\left(L^{k}\right)$ for $1 \leq i \leq p+1$ and $k \geq 1$ are injective.

\section{The Ideal of $X$ in $\mathbb{P}_{N}$}

As before, let $X$ be a projective variety defined over an algebraically closed field $K, L$ an ample line bundle on $X$ and $V \subseteq H^{0}(L)$ a subvector space. Suppose $V$ satisfies property $\widetilde{N}_{0}$. Then according to Proposition $1.1 \mathrm{~V}$ defines an embedding $\varphi_{V}: X \hookrightarrow \mathbb{P}_{N}$. The aim of this section is to give a criterium for the homogeneous ideal $I=I_{X / \mathbb{P}_{N}}$ of $X$ in $\mathbb{P}_{N}$ to be generated by forms of degree $\leq k$.

With the notation as in the previous section, there is an exact sequence of $S_{-}$ modules

$$
0 \rightarrow I \rightarrow S \rightarrow R \rightarrow 0
$$


In fact, by definition $I$ is the kernel of the canonical map $S \rightarrow R$, and surjectivity follows from the assumption that $V$ satisfies $\widetilde{N}_{0}$. Combining this with the minimal free resolution 2(1) of $R$, one obtains a resolution of the graded $S$-module $I$

$$
0 \rightarrow \bigoplus_{j} S(-j)^{\tilde{b}_{r j}} \rightarrow \ldots \rightarrow \bigoplus_{j} S(-j)^{\tilde{b}_{1 j}} \rightarrow I \rightarrow 0 .
$$

For an integer $k \geq 2$ the ideal $I$ is said to be generated by forms of degree $\leq k$, if $\tilde{b}_{1 j}=0$ for $j \geq k+1$, i.e. there exists a surjective homomorphism of graded $S$-modules

$$
\bigoplus_{j=2}^{k} S(-j)^{\tilde{b}_{1 j}} \rightarrow I \rightarrow 0 .
$$

(3.1) Theorem. Suppose $V \subseteq H^{0}(L)$ is a subvector space satisfying property $\widetilde{N}_{0}$. If $H^{1}\left(\bigwedge^{2} M_{V} \otimes L^{j}\right)=0$ for $j \geq k-1$, then the ideal $I_{X / \mathbb{P}_{N}}$ is generated by forms of degree $\leq k$.

Proof. Since $\operatorname{dim} \mathcal{K}_{i, j}(V, R)=\tilde{b}_{i, i+j}$, it suffices to show that $\mathcal{K}_{1, j}(V, R)=0$ for $j \geq k$. Consider the following diagram:

$$
\begin{aligned}
& 0 \\
& \downarrow \\
& H^{0}\left(\stackrel{2}{\bigwedge} M_{V} \otimes L^{j-1}\right) \\
& \bigwedge^{2} V \otimes H^{0}\left(L^{j-1}\right) \quad \rightarrow V \otimes H^{0}\left(L^{j}\right) \quad \rightarrow H^{0}\left(L^{j+1}\right) \quad \rightarrow 0 \\
& \psi \downarrow \\
& 0 \rightarrow \quad H^{0}\left(M_{V} \otimes L^{j}\right) \quad \rightarrow V \otimes H^{0}\left(L^{j}\right) \quad \rightarrow H^{0}\left(L^{j+1}\right) \quad \rightarrow 0 \\
& H^{1}\left(\bigwedge^{2} M_{V} \otimes L^{j-1}\right)
\end{aligned}
$$

For $j \geq 3$ the upper horizontal sequence is the Koszul complex $\left(K_{\bullet} \otimes R\right)_{1+j}$. The lower sequence is the exact cohomology sequence of $0 \rightarrow M_{V} \otimes L^{j} \rightarrow V \otimes L^{j} \rightarrow$ $L^{j+1} \rightarrow 0$. By assumption $\psi$ is surjective for $j \geq k$. Thus the Koszul sequence is exact at $V \otimes H^{0}\left(L^{j}\right)$, i.e. $\mathcal{K}_{1, j}(V, R)=0$, for $j \geq \max (k, 3)$. This proves the assertion for $k \geq 3$. Now suppose $j=k=2$. As in the proof of Theorem 2.4, the 2-normality property of $V$ implies that the sequence $\bigwedge^{2} \otimes R_{1} \rightarrow V \otimes R_{2} \rightarrow R_{3}$ $\rightarrow 0$ is exact, i.e. $\mathcal{K}_{1,2}(V, R)=0$.

The same proof yields

(3.2) Remark. The ideal $I_{X / \mathbb{P}_{N}}$ is generated by forms of degree $\leq k$ if and only if the maps $H^{1}\left(\bigwedge^{2} M_{V} \otimes L^{j}\right) \rightarrow \bigwedge^{2} V \otimes H^{1}\left(L^{j}\right)$ are injective for all $j \geq k-1$.

In the following 4 sections the results presented so far will be applied for noncomplete linear systems on complex abelian varieties.

\section{2-Normal Linear Systems on Elliptic Curves and General Abelian Varieties}

Throughout the following 4 sections, dealing with linear systems on abelian varieties, the field of definition will be exclusively the field of complex numbers $\mathbb{C}$. 
Let $(X, M)$ be a polarized elliptic curve. The vector space $H^{0}(M)$ is an irreducible representation of the theta group $\mathcal{G}(M)$ associated with the line bundle $M$. The aim of this section is to study 2-normality of those subvector spaces $V \subseteq H^{0}(M)$ admitting a $\mathcal{G}(M)$-action. By semicontinuity one obtains in this way results on 2-normality for a general subvector space of $H^{0}(M)$ of small codimension.

Suppose the line bundle $M$ is of degree $d$. Associated with $M$ is an isogeny

$$
\phi_{M}: X \rightarrow \widehat{X}, \quad x \mapsto t_{x}^{*} M \otimes M^{-1},
$$

where $\widehat{X}$ denotes the dual elliptic curve and $t_{x}$ the translation map $y \mapsto x+y$ on $X$. The kernel of $M$ is

$$
K(M) \simeq \mathbb{Z} / d \mathbb{Z} \times \mathbb{Z} / d \mathbb{Z}
$$

The theta group $\mathcal{G}(M)$ is a central extension of $K(M)$ by $\mathbb{C}^{*}$ :

$$
0 \rightarrow \mathbb{C}^{*} \rightarrow \mathcal{G}(M) \stackrel{\pi}{\longrightarrow} K(M) \rightarrow 0 .
$$

Its commutator map is a nondegenerate, alternating bilinear form $e^{M}: K(M) \times$ $K(M) \rightarrow \mathbb{C}^{*}$. Choose a decomposition

$$
K(M)=K_{1} \oplus K_{2}
$$

into cyclic subgroups $K_{i} \simeq \mathbb{Z} / d \mathbb{Z}$ with respect to $e^{M}$ (see [CAV], Theorem 6.3.4). Let $\mathcal{G}_{2} \subset \mathcal{G}(M)$ be a section over $K_{2}$, i.e. a subgroup of $\mathcal{G}(M)$ isomorphic to $K_{2}$ via $\pi$. $\mathcal{G}_{2}$ being abelian, the $\mathcal{G}_{2}$-module $H^{0}(M)$ splits into one-dimensional eigenspaces

$$
H^{0}(M)=\bigoplus_{\chi \in \mathcal{G}_{2}^{*}} U_{\chi}
$$

with $\mathcal{G}_{2}^{*}=\operatorname{Hom}\left(\mathcal{G}_{2}, \mathbb{C}^{*}\right)$, the character group of $\mathcal{G}_{2}$. Notice that ord $\mathcal{G}_{2}^{*}=\operatorname{ord} K_{2}=$ $d=h^{0}(M)$, and that $\mathcal{G}_{2}^{*} \simeq K_{2}^{*} \simeq K_{1}$, according to loc. cit. In the sequel $\mathcal{G}_{2}^{*}$ and $K_{1}$ will be identified. For a subset $S \subseteq K_{1}$ denote

$$
V_{S}:=\bigoplus_{x \in S} U_{x} \subset H^{0}(M)
$$

Obviously the vector spaces $V_{S}$ are exactly the subvector spaces of $H^{0}(M)$ invariant with respect to the action of $\mathcal{G}_{2}$. The aim of this section is to prove the following.

(4.1) Theorem. Suppose $M=L^{2}$ is the square of an ample line bundle $L$ on $X$ and $S \subset K_{1}$ is a subset satisfying

$$
\text { for all } y \in K_{1} \text { there exists an } x \in(y-2 S) \cap(2 S-y) \text { different }
$$

from $z_{0}$, the non-trivial 2-division point of $K_{1}$.

Then $V_{S}$ is 2-normal.

It can easily be checked that the condition (*) is fulfilled if $d=\operatorname{deg} M \geq 6$ is even and $S=K_{1}-\{0\}$. Since moreover 2-normality is an open condition for the subvector spaces of $H^{0}(M)$ of codimension 1 , this implies, together with Corollary 1.6:

(4.2) Corollary. Suppose $X$ is an elliptic curve and $M \in \operatorname{Pic}(X)$ a line bundle of degree $2 n \geq 6$. The general subvector space $V$ of $H^{0}(M)$ of codimension 1 satisfies property $\widetilde{N}_{0}$.

For general polarized abelian varieties this yields 
(4.3) Corollary. Suppose $(X, L)$ is a general polarized abelian variety of type $\left(d_{1}, \ldots d_{g}\right)$. Let $n \geq 3$ be an integer such that $n d_{g} \geq 6$ is even, and let $c \leq n^{g-1}$. Then the general subvector space $V \subseteq H^{0}\left(L^{n}\right)$ of codimension c is 2-normal.

Proof. By semicontinuity it suffices to consider the case $(X, L)=\left(X_{1}, L_{1}\right) \times\left(X_{2}, L_{2}\right)$ with a polarized abelian variety $\left(X_{1}, L_{1}\right)$ of type $\left(d_{1}, \ldots, d_{g-1}\right)$ and a polarized elliptic curve $X_{2}$ of type $\left(d_{g}\right)$. According to Corollary $4.2, H^{0}\left(X_{2}, L_{2}^{n}\right)$ contains a 2 -normal subvector space $V_{2}$ of codimension 1 . Then the space

$$
V:=p_{X_{1}}^{*} H^{0}\left(X_{1}, L_{1}^{n}\right) \otimes p_{X_{2}}^{*} V_{2} \subseteq H^{0}\left(X, L^{n}\right)
$$

is of codimension

$$
c=\frac{h^{0}\left(L^{n}\right)}{n d_{g}} \geq n^{g-1} .
$$

By [CAV], Theorem 7.3.1, $H^{0}\left(X_{1}, L_{1}^{n}\right)$ is 2 -normal. Hence by the Künneth isomorphism $V$ is $2-$ normal as well. As above, this implies the assertion.

The idea of the proof of Theorem 4.1 is to restrict the Theta Multiplication Formula to the subspaces $V_{S}$. For this we need some notation: Suppose $X=\mathbb{C} / \Lambda$ with a lattice $\Lambda \subset \mathbb{C}$. In the proofs we do not distinguish between points on $X$ and their representatives in $\mathbb{C}$. Consider the sections of line bundles on $X$ as canonical theta functions on $\mathbb{C}$ (see $[\mathrm{CAV}]$, Section 3.2). For all $x \in X$ the following diagram is commutative:

$$
\begin{array}{ccc}
H^{0}(M) \otimes H^{0}(M) & \longrightarrow & H^{0}\left(M^{2}\right) \\
\tau_{x} \otimes \tau_{x} \downarrow & & \downarrow \tau_{x} \\
H^{0}\left(t_{x}^{*} M\right) \otimes H^{0}\left(t_{x}^{*} M\right) & \longrightarrow & H^{0}\left(t_{x}^{*} M^{2}\right)
\end{array}
$$

where $\tau_{x}$ denotes the homomorphism induced by the translation $t_{x}$ (see [CAV], Corollary 3.2.9). Hence without loss of generality we may assume that the line bundle $M$ is of characteristic 0 with respect to the decomposition (1) (in the sense of [CAV], Section 3.1), and [CAV], Theorem 3.2.7, yields a basis

$$
\left\{\vartheta_{x}^{M} \mid x \in K_{1}\right\} \subset H^{0}(M) .
$$

In particular,

$$
V_{S}=\operatorname{span}\left\{\vartheta_{x}^{M} \mid x \in S\right\} .
$$

Recall that the group $K\left(M^{2}\right)=\operatorname{ker} \phi_{M^{2}}$ satisfies $2 K\left(M^{2}\right)=K(M)$. Let

$$
K\left(M^{2}\right)=K\left(M^{2}\right)_{1} \oplus K\left(M^{2}\right)_{2}
$$

be a decomposition compatible with (1), i.e. $2 K\left(M^{2}\right)_{i}=K_{i}$ for $i=1,2$, and let

$$
\left\{\vartheta_{x}^{M^{2}} \mid x \in K\left(M^{2}\right)_{1}\right\} \subset H^{0}\left(M^{2}\right)
$$

be the corresponding basis. Denote by $Z_{2}:=K_{1} \cap X_{2}=\left\{0, z_{0}\right\}$ the subgroup of 2 -division points of $K_{1}$, and $\widehat{Z}_{2}=\operatorname{Hom}\left(Z_{2}, \mathbb{C}^{*}\right)$ its character group. Then the canonical theta functions

$$
\begin{array}{ll}
\theta_{\left(x_{1}, x_{2}\right), \rho}^{M}:=\sum_{z \in Z_{2}} \rho(z) \vartheta_{x_{1}+z}^{M} \otimes \vartheta_{x_{2}+z}^{M}, & \left(x_{1}, x_{2}\right) \in K_{1}^{2}, \rho \in \widehat{Z}_{2}, \\
\theta_{y, \rho}^{M^{2}}:=\sum_{z \in Z_{2}} \rho(z) \vartheta_{y+z}^{M^{2}}, & y \in K\left(M^{2}\right)_{1}, \rho \in \widehat{Z}_{2},
\end{array}
$$

generate the vector spaces $H^{0}(M) \otimes H^{0}(M)$ and $H^{0}\left(M^{2}\right)$ respectively (see [CAV], Section 7.1). In terms of these theta functions the multiplication map $\mu: H^{0}(M) \otimes$ $H^{0}(M) \rightarrow H^{0}\left(M^{2}\right)$ gives 
(4.4) Lemma. For all $x_{1}, x_{2} \in K_{1}$ and $y_{1}, y_{2} \in K\left(M^{2}\right)_{1}$ satisfying $y_{1}+y_{2}=x_{1}$ and $y_{1}-y_{2}=x_{2}$, and for all $\rho \in \widehat{Z}_{2}$,

$$
\mu\left(\theta_{\left(x_{1}, x_{2}\right), \rho}^{M}\right)=\theta_{y_{2}, \rho}^{M^{2}}(0) \theta_{y_{1}, \rho}^{M^{2}} .
$$

For a proof see $[\mathrm{CAV}]$, Corollary 7.1.4. For $y \in K_{1}$ define the set $S_{y}:=$ $\bigcap_{z \in Z_{2}}(z-S) \cap(z-y+S)$. An immediate consequence of Lemma 4.4 is

(4.5) Lemma. If for all $y \in K\left(M^{2}\right)_{1}$ and $\rho \in \widehat{Z}_{2}$ there exists an $x \in S_{2 y} \subseteq K_{1}$ such that

$$
\theta_{y+x, \rho}^{M^{2}}(0) \neq 0
$$

then $V_{S}$ is 2-normal.

Proof. According to Lemma 4.4, $\mu\left(\theta_{(2 y+x,-x), \rho}^{M}\right)=\theta_{y+x, \rho}^{M^{2}}(0) \cdot \theta_{y, \rho}^{M^{2}}$. Hence $\theta_{y, \rho}^{M^{2}} \in$ $\operatorname{im}\left\{V_{S} \otimes V_{S} \rightarrow H^{0}\left(M^{2}\right)\right\}$, if $\theta_{y+x, \rho}^{M^{2}}(0) \neq 0$ and $\theta_{(2 y+x,-x), \rho}^{M} \in V_{S} \otimes V_{S}$. The latter condition certainly holds if $x \in(z-S) \cap(z-2 y+S)$ for all $z \in Z_{2}$, i.e. $x \in S_{2 y}$.

For $y \in K\left(M^{2}\right)_{1}$ and $\rho \in \widehat{Z}_{2}$ define the subvector space

$$
H_{y, \rho}:=\operatorname{span}\left\{\theta_{y+x, \rho}^{M^{2}} \mid x \in S_{2 y}\right\} \subseteq H^{0}\left(M^{2}\right) .
$$

If $S_{2 y} \neq 0, S_{2 y}$ is invariant with respect to translations by elements of $Z_{2}$ and

$$
\operatorname{dim} H_{y, \rho}=\# S_{2 y} / Z_{2}
$$

The decomposition (1) induces a decomposition $K(L)_{1} \oplus K(L)_{2}$ of $K(L)=2 K(M)$. Let $\left\{\vartheta_{x}^{L} \mid x \in K(L)_{1}\right\} \subset H^{0}(L)$ be the associated basis. The subvector space

$$
W_{y}:=\operatorname{span}\left\{\vartheta_{2 x}^{L} \mid x \in S_{2 y}\right\} \subset H^{0}(L) .
$$

is of the same dimension as $H_{y, \rho}$. For all $y \in K\left(M^{2}\right)$ there are isomorphisms $2^{*} t_{2 y}^{*} L \simeq t_{y}^{*} 2^{*} L \simeq M^{2}$. Hence the holomorphic map $t_{2 y} \circ 2: X \rightarrow X$ induces an embedding $2^{*} \tau_{2 y}: H^{0}(L) \hookrightarrow H^{0}\left(M^{2}\right)$.

(4.6) Lemma. For $\rho \in \widehat{Z}_{2}$ define $s \in K\left(M^{2}\right)_{2}$ by $\rho=e^{M^{2}}(s, \cdot)$. Then for all $y \in K\left(M^{2}\right)_{1}$

$$
2^{*} \tau_{2(y+s)} W_{y}=H_{y, \rho} .
$$


Proof. Let $x \in K_{1}$, and let $H=c_{1}(L)$ be the hermitian form associated with $L$. $2^{*} \tau_{2(y+s)} \vartheta_{2 x}^{L}=2^{*}\left[e(-2 \pi H(\cdot, y+s)-2 \pi H(y+s, y+s)) \vartheta_{2 x}^{L}(\cdot+2(y+s))\right]$

(by [CAV], Corollary 3.2.9)

$$
=e(-4 \pi H(\cdot, y+s)-2 \pi H(y+s, y+s)) \sum_{z^{\prime} \in Z_{2}} \vartheta_{x+z^{\prime}}^{M^{2}}(\cdot+y+s)
$$

(by the Isogeny Theorem, [CAV], p. 160)

$$
=e(2 \pi H(y+s, y+s))[1,-y-s] \sum_{z^{\prime} \in Z_{2}} \vartheta_{x+z^{\prime}}^{M^{2}}
$$

(by [CAV], 6.4(1), since $c_{1}\left(M^{2}\right)=4 H$ )

$$
=e(2 \pi H(y+s, y+s)) a_{M^{2}}(-y-s, 0)^{-1} \sum_{z^{\prime} \in Z_{2}} e^{M^{2}}\left(x+z^{\prime}+y,-s\right) \vartheta_{x+z^{\prime}+y}^{M^{2}}
$$

(by $[\mathrm{CAV}]$, Proposition 6.4.2)

$$
=k \sum_{z^{\prime} \in Z_{2}} \rho\left(z^{\prime}\right) \vartheta_{x+z^{\prime}+y}^{M^{2}}=k \theta_{x+y, \rho}^{M^{2}}
$$

with some constant $k \neq 0$. This shows that $2^{*} \tau_{2(y+s)} \vartheta_{2 x}^{L} \in H_{y, s}$ if and only if $x \in S_{2 y}$. Since $\operatorname{dim} H_{y, s}=\operatorname{dim} W_{y}$ and $2^{*} \tau_{2(y+s)}$ is injective, this implies the assertion.

Proof of Theorem 4.1. According to Lemmas 4.5 and 4.6 it suffices to show that for all $y \in K\left(M^{2}\right)_{1}$ and $\rho=e^{M^{2}}(s, \cdot) \in \widehat{Z}_{2}$ the point $2 y+2 s$ is no base point of the subvector space $W_{y} \subseteq H^{0}(L)$. The assumption (*) says that

$$
2 S_{2 y}+2 y=(2 y-2 S) \cap(2 S-2 y) \neq \emptyset \text { and } \neq\left\{z_{0}\right\}
$$

In particular, $S_{2 y} \neq 0$; hence $W_{y} \neq \emptyset$ and it suffices to show that $\vartheta_{2 x}^{L}(2 y+2 s) \neq 0$ for some $x \in S_{2 y}$, or equivalently $\vartheta_{0}^{L}(2 x+2 y+2 s) \neq 0$ (see [CAV], 3.2(4)). Let $m$ and $n$ by generators of $K(M)_{1} \simeq \mathbb{Z} / d \mathbb{Z}$ and $K(M)_{2} \simeq \mathbb{Z} / d \mathbb{Z}$ respectively. The theta function $\vartheta_{0}^{L} \in H^{0}(E, L)$ is the pull-back of the canonical theta function of characteristic 0 via the degree $\frac{d}{2}$-isogeny $\pi: E \rightarrow F:=E /\langle 2 n\rangle$. The corresponding divisor on $F$ (of degree 1) has support $\pi\left(\frac{d}{2} m+n\right)=\pi\left(z_{0}+n\right) \in F$ (notice that $d$ is even, since $\left.L^{2}=M\right)$. Hence the support of the divisor defined by $\vartheta_{0}^{L}$ on $E$ is

$$
\operatorname{supp}\left(\vartheta_{0}^{L}\right)=\left\{\left(z_{0}+n\right),\left(z_{0}+3 n\right), \ldots,\left(z_{0}+(d-1) n\right)\right\} .
$$

Hence it suffices to show that for all $y$ and $s$ as above

$$
\left\{2 x+2 y+2 s \mid x \in S_{2 y}\right\} \not \subset \operatorname{supp}\left(\vartheta^{L}\right) .
$$

But by (2) there exists an $x \in S_{2 y}$ with $2 x+2 y \neq z_{0}$. This shows $(*)$.

\section{The Ideal of an Abelian Surface in $\mathbb{P}_{N}$}

Let $A$ be an abelian surface and $L$ an ample line bundle on $A$. In this section we will study the property $\widetilde{N}_{0}$ for $2-$ normal subvector spaces of $H^{0}(L)$. Moreover we give a criterion for the ideal of $A$ in $\mathbb{P}_{N}$ to be generated by quadrics and cubics. This result seems to be new even in the case of complete linear systems. 
(5.1) Theorem. Suppose $V \subseteq H^{0}(L)$ is a 2-normal, base point free subvector space of codimension c. If

$$
h^{0}(L) \geq 3 c+8
$$

then $V$ satisfies property $\widetilde{N}_{0}$.

For the proof we need the following well-known lemma.

(5.2) Lemma. Let $X \subset \mathbb{P}_{N}$ be a nondegenerate, irreducible, reduced proper subvariety of dimension $n \geq 1$. The general $(N-n)$-secant $(N-n-1)$-plane of $X$ is no multisecant.

For $n=1$ and $N \leq 3$, see [Ha], IV 3 . The general case follows by induction using central projections and Bertini's theorem.

Proof of Theorem 5.1. The polarized abelian surface $(A, L)$ is not the polarized product of elliptic curves, $V$ (and hence also $L$ ) being base point free (see [CAV], Lemma 10.1.1). According to $[\mathrm{CAV}]$, Lemma 10.1.2 and Theorem 4.5.5, $L^{2}$ is very ample and thus $\varphi_{V}: A \hookrightarrow \mathbb{P}_{N}=\mathbb{P}\left(V^{*}\right)$, with $N=h^{0}(L)-c-1$, is an embedding by Proposition 1.1. According to Lemma 1.3 and Corollary 1.5 it suffices to show that

$$
H^{1}\left(M_{V} \otimes L^{2}\right)=0 .
$$

Choose points $p_{1}, \ldots, p_{N-2} \in A \subset \mathbb{P}_{N}$ in general position. According to the above lemma we may assume that the $(N-3)$-plane $P_{N-3}=\operatorname{span}\left\{p_{1}, \ldots, p_{N-2}\right\} \subset \mathbb{P}_{N}$ is no multisecant of $A$. To $P_{N-3} \subset \mathbb{P}\left(V^{*}\right)$ corresponds a 3-dimensional subvector space $W_{1} \subset V$. Denote by

$$
f_{1}: Y_{1} \rightarrow A
$$

the blow-up of $A$ in the points $p_{1}, \ldots, p_{N-2}$ and by $E_{1}, \ldots, E_{N-2}$ the exceptional divisors. The evaluation map $W_{1} \otimes \mathcal{O}_{Y_{1}} \rightarrow f_{1}^{*} L\left(-\sum_{j=1}^{N-2} E_{j}\right)$ is surjective, $P_{N-3}$ being no multisecant. Consider the following commutative diagram with exact rows and columns and a rank 2 vector bundle $\mathcal{F}$ on $Y_{1}$ :

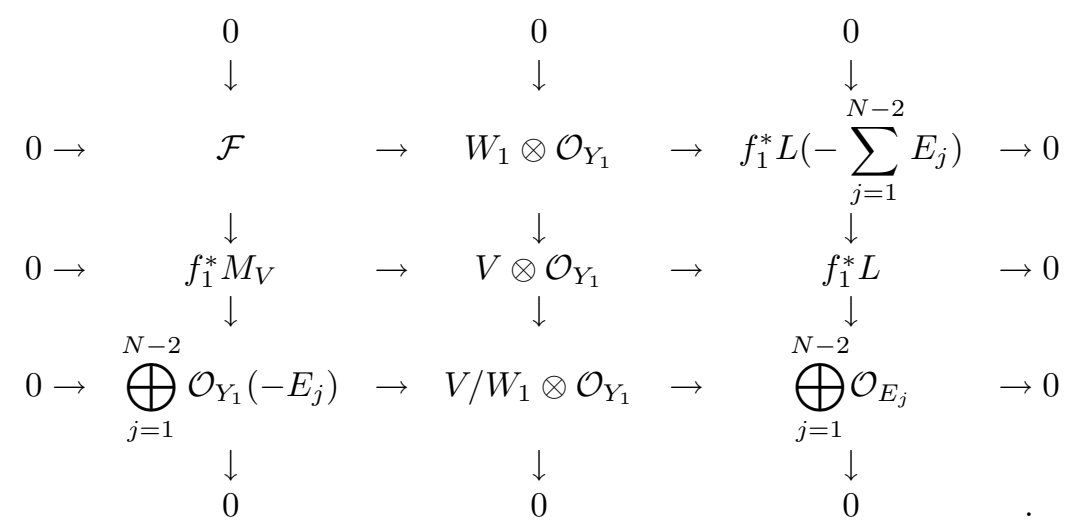

Choose a general $(N-2)$-plane $P_{N-2} \subset \mathbb{P}_{N}$ containing $P_{N-3} . P_{N-2}$ intersects the abelian surface $A$ in $\delta:=\operatorname{deg}_{\mathbb{P}_{N}} A=2 h^{0}(L)$ distinct points $p_{1}, \ldots, p_{N-2}, p_{N-1}, \ldots$, 
$p_{\delta}$. Let $W_{2} \subset W_{1}$ be the 2-dimensional subvector space corresponding to $P_{N-2}$. Moreover, denote by

$$
f_{2}: Y_{2} \rightarrow Y_{1}
$$

the blow up of $Y_{1}$ in the $\delta-N+2$ points $f_{1}^{-1}\left(p_{N-1}\right), \ldots, f_{1}^{-1}\left(p_{\delta}\right)$, and by $E_{N-1}, \ldots$, $E_{\delta}$ the exceptional divisors. By abuse of notation denote by $E_{1}, \ldots, E_{N-2}$ the preimages of the exceptional divisors of $f_{1}$ as well. Setting $f:=f_{1} \circ f_{2}$ as above, we see that the evaluation map $W_{2} \otimes \mathcal{O}_{Y_{2}} \rightarrow f^{*} L\left(-\sum_{j=1}^{\delta} E_{j}\right)$ is surjective and the following diagram is commutative with exact rows and columns:

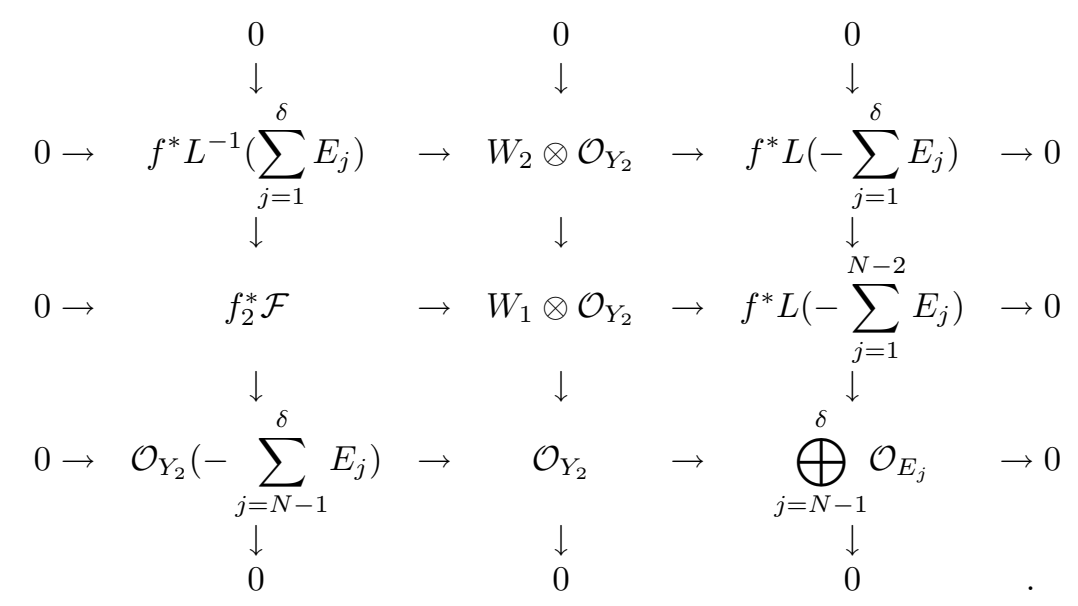

Using the left-hand vertical sequences in (2) and (3), the vanishing (1) follows from the following 3 vanishings:

a) $h^{1}\left(f_{1}^{*} L^{2}\left(-E_{j}\right)\right)=0$ for $1 \leq j \leq N-2$,

b) $h^{1}\left(f^{*} L\left(\sum_{j=1}^{\delta} E_{j}\right)\right)=0$, and

c) $h^{1}\left(f^{*} L^{2}\left(-\sum_{j=N-1}^{\delta} E_{j}\right)\right)=0$.

As for a), denoting by $I_{p_{j}}$ the ideal sheaf of $p_{j}$ on $A$, the projection formula implies $h^{1}\left(f_{1}^{*} L^{2}\left(-E_{j}\right)\right)=h^{1}\left(L^{2} \otimes I_{p_{j}}\right)$. Since $L^{2}$ is base point free, $H^{0}\left(L^{2}\right) \rightarrow$ $H^{0}\left(L^{2} \mid p_{j}\right)$ is surjective and hence $h^{1}\left(L^{2} \otimes I_{p_{j}}\right) \leq h^{1}\left(L^{2}\right)=0$.

As for b), Serre duality and the projection formula imply

$$
H^{1}\left(f^{*} L\left(\sum_{j=1}^{\delta} E_{j}\right)\right)=H^{1}\left(f^{*} L \otimes \omega_{Y_{2}}\right)=H^{1}\left(f^{*} L^{-1}\right)=H^{1}\left(L^{-1}\right)=0 .
$$

As for c), denote $Z=\left\{p_{N-1}, \ldots, p_{\delta}\right\}$. It suffices to show that $h^{1}\left(L^{2} \otimes I_{Z}\right)=0$, or equivalently that $H^{0}\left(L^{2}\right) \rightarrow H^{0}\left(L^{2} \mid Z\right)=h^{0}\left(\mathcal{O}_{Z}\right)$ is surjective. Consider the following commutative diagram:

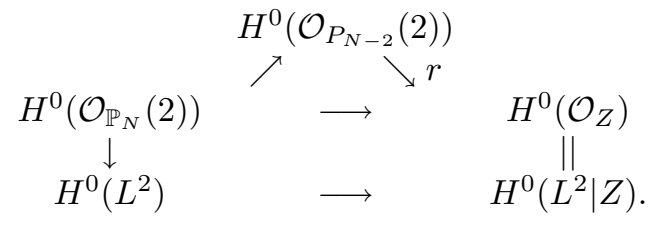

It suffices to show that $r$ is surjective. Castelnuovo's lemma implies that $2 n+1$ points in general position impose independent conditions on quadrics in $\mathbb{P}_{n}$. Hence 
$r$ is surjective if $\# Z\left(=2 h^{0}(L)-N+2\right) \leq 2(N-2)+1$ or equivalently if $h^{0}(L) \geq$ $3 c+8$.

Using the same methods one can derive a numerical condition for the ideal $I(V)$ of $A$ in $\mathbb{P}_{N}$ to be generated by forms of degree $\leq 3$.

(5.3) Theorem. Suppose $V \subseteq H^{0}(L)$ is a subvector space of codimension c satisfying property $\widetilde{N}_{0}$. If

$$
h^{0}(L) \geq 3 c+9
$$

then the ideal $I(V)$ is generated by quadrics and cubics.

For the proof we need the following well-known result on exterior products of exact sequences of vector bundles.

(5.4) Proposition. Suppose $0 \rightarrow E \rightarrow F \rightarrow G \rightarrow 0$ is an exact sequence of vector bundles on a projective variety $X$. Then there exists a vector bundle $H$ on $X$ fitting into the following exact sequences:

$$
\begin{aligned}
& 0 \rightarrow \bigwedge^{2} E \rightarrow \rightarrow \quad H \quad \rightarrow E \otimes G \rightarrow 0 \\
& 0 \rightarrow H \rightarrow \bigwedge^{2} F \rightarrow \bigwedge^{2} G \rightarrow 0
\end{aligned}
$$

Proof of Theorem 5.3. According to Theorem 3.1 it suffices to show that

$$
H^{1}\left(\bigwedge^{2} M_{V} \otimes L^{k}\right)=0
$$

for $k \geq 2$. Let the notation be as in the proof of Theorem 5.1. Applying Proposition 5.4 to the left-hand vertical sequence of diagram (2) yields

$$
\begin{gathered}
0 \rightarrow \bigwedge^{2} \mathcal{F} \rightarrow H \rightarrow \mathcal{F} \otimes\left(\bigoplus_{j=1}^{N-2} \mathcal{O}_{Y_{1}}\left(-E_{j}\right)\right) \rightarrow 0 \\
0 \rightarrow H \rightarrow \bigwedge^{2} f_{1}^{*} M_{V} \rightarrow \bigwedge^{2}\left(\bigoplus_{j=1}^{N-2} \mathcal{O}_{Y_{1}}\left(-E_{j}\right)\right) \rightarrow 0
\end{gathered}
$$

with a vector bundle $H$ on $A$. Moreover, recall the exact sequence

$$
0 \rightarrow f^{*} L^{-1}\left(\sum_{j=1}^{\delta} E_{j}\right) \rightarrow f_{2}^{*} \mathcal{F} \rightarrow \mathcal{O}_{Y_{2}}\left(-\sum_{j=N-1}^{\delta} E_{j}\right) \rightarrow 0
$$

of diagram (3). It suffices to show that (for all $k \geq 2$ )

a) $H^{1}\left(\bigwedge^{2}\left(\oplus_{j=1}^{N-2} \mathcal{O}_{Y_{1}}\left(-E_{j}\right) \otimes f_{1}^{*} L^{k}\right)\right)=0$,

b) $H^{1}\left(\bigwedge^{2} \mathcal{F} \otimes f_{1}^{*} L^{k}\right)=0$,

c) $H^{1}\left(\oplus_{j=1}^{N-2} \mathcal{O}_{Y_{2}}\left(-E_{j}\right) \otimes f^{*} L^{k-1}\left(\sum_{j=1}^{\delta} E_{j}\right)\right)=0$,

d) $H^{1}\left(\oplus_{j=1}^{N-2} \mathcal{O}_{Y_{2}}\left(-E_{j}\right) \otimes f^{*} L^{k}\left(-\sum_{j=N-1}^{\delta} E_{j}\right)\right)=0$.

As for a), for $1 \leq i<j \leq N-2$ we have

$$
H^{1}\left(f_{1} L^{k}\left(-E_{i}-E_{j}\right)\right)=H^{1}\left(L^{k} \otimes I_{p_{i}+p_{j}}\right)=\operatorname{coker}\left(H^{0}\left(L^{k}\right) \rightarrow H^{0}\left(\left.L^{k}\right|_{p_{i}+p_{j}}\right)\right) .
$$

Since $L^{k}$ is very ample the right-hand side is 0 , and this implies a).

As for b), sequence (6), the projection formula and Serre duality imply

$$
\begin{aligned}
H^{1}\left(\bigwedge^{2} \mathcal{F} \otimes f_{1}^{*} L^{k}\right) & =H^{1}\left(\bigwedge^{2} f_{2}^{*} \mathcal{F} \otimes f^{*} L^{k}\right)=H^{1}\left(f^{*} L^{k-1}\left(\sum_{j=1}^{N-2} E_{j}\right)\right) \\
& =H^{1}\left(f_{1}^{*} L^{k-1} \otimes \omega_{Y_{1}}\right)=H^{1}\left(f_{1}^{*} L^{1-k}\right)=H^{1}\left(L^{1-k}\right)=0 .
\end{aligned}
$$

Statement c) is a consequence of

$$
\begin{aligned}
H^{1}\left(f^{*} L^{k-1}\left(\sum_{j=1}^{\delta} E_{j}-E_{i}\right)\right) & =H^{1}\left(f^{*} L^{k-1}\left(-E_{i}\right) \otimes \omega_{Y_{2}}\right) \\
& =H^{1}\left(f^{*} L^{1-k}\left(E_{i}\right)\right)=H^{1}\left(L^{1-k}\right)=0
\end{aligned}
$$

for $1 \leq i \leq N-2$. 
As for d), it suffices to show that for all $k \geq 2$

$$
H^{1}\left(f^{*} L^{k}\left(-E_{i}-\sum_{j=N-1}^{\delta} E_{j}\right)\right)=H^{1}\left(L^{k} \otimes I_{Z}\right)=0
$$

with $1 \leq i \leq N-2$ and $Z=\left\{p_{i}, p_{N-1}, \ldots, p_{\delta}\right\}$. With the same reasoning as in the proof of Theorem 5.1 the above cohomology group vanishes if $\# Z \leq 2(N-2)+1$ or, equivalently, $h^{0}(L) \geq 3 c+9$.

\section{The Property $\tilde{N}_{p}$ For Linear systems on General Abelian Varieties}

Let $(X, L)$ be a general abelian variety of dimension $g$ and $V \subseteq H^{0}(L)$ a general subvector space. The aim of this section is to prove a criterion for $V$ to satisfy the property $\widetilde{N}_{p}$. For this it is convenient to consider $L$ as a power of an ample line bundle $M$ on $X$, i.e. $L=M^{n}$ for some $n \geq 1$. If $M$ is of type $\left(d_{1}, \ldots, d_{g}\right)$, then we have

(6.1) Theorem. Suppose $V \subseteq H^{0}(L)$ is a general subvector space of codimension c and $p$ is an integer $\geq 0$. If the integers $d_{g}, n$ and $c$ satisfy the following 3 numerical conditions

a) $n \geq \max \left(k_{0}(p+1), 3\right)$ with $k_{0}=\max \left(2,\left\lceil 4 / d_{g}\right\rceil\right)$,

b) $n d_{g} \geq 6$ and is even,

c) $c \leq n^{g-1}$,

then $V$ satisfies property $\widetilde{N}_{p}$.

(Here $\lceil x\rceil$ denotes the least integer $\geq x$.)

An immediate consequence is the following analogue to Kempf's result on complete linear systems on abelian varieties (see $[\mathrm{K}]$ ).

(6.2) Corollary. Let $(X, L)$ be a general polarized abelian variety of type $\left(d_{1}, \ldots, d_{g}\right), d_{g} \geq 2$. For integers $p \geq 1, n \geq 2 p+2$ with $n d_{g} \equiv 0(\bmod 2)$, and $c \leq n^{g-1}$ the general subvector space $V \subseteq H^{0}(L)$ of codimension $c$ satisfies property $\widetilde{N}_{p}$.

(6.3) Remark. In the following version of Theorem 6.1 the assumption "general" for $V$ can be omitted:

Under the hypotheses $6.1 \mathrm{a}), \mathrm{b})$, and c) any 2-normal subvector space $V \subseteq$ $H^{0}(L)$ of codimension $c$ satisfying

$$
H^{i}\left(M_{V} \otimes M^{k} \otimes P\right)=0
$$

for $i \geq 1, k \geq k_{0}$ and $P \in \operatorname{Pic}^{0}(X)$ satisfies property $\widetilde{N}_{p}$.

For the proofs of Theorem 6.1 and Remark 6.3 we need the following lemmas.

(6.4) Lemma. Let $(E, M)$ be a polarized elliptic curve with $d=\operatorname{deg} M$. Suppose $n \geq 1$ such that $n d \geq 6$ is even. There exists a subvector space $W \subseteq H^{0}\left(E, M^{n}\right)$ of codimension 1 satisfying $\widetilde{N}_{p}$, such that the canonical map

$$
W \otimes H^{0}\left(M^{k} \otimes P\right) \rightarrow\left(M^{n+k} \otimes P\right)
$$

is surjective, for all $k$ with $d k>3$ and all $P \in \operatorname{Pic}^{0}(E)$. 
Proof. According to Corollary 4.2 there exists a subvector space $W \subset H^{0}\left(E, M^{n}\right)$ of codimension 1 satisfying $\widetilde{N}_{0}$. It suffices to show that

$$
H^{1}\left(M_{W} \otimes M^{k} \otimes P\right)=0
$$

with $M_{W}$ the vector bundle on $E$ associated with $W$. According to Proposition 1.1 $W$ is very ample, i.e. $W$ defines an embedding $\varphi_{W}: E \hookrightarrow \mathbb{P}_{N}, N=n d-2$. Choose points $p_{1}, \ldots, p_{N-1} \in E \subset \mathbb{P}_{N}$ in general position. According to Lemma 5.2 we may assume that the $(N-2)$-plane $P_{N-2}=\operatorname{span}\left\{p_{1}, \ldots, p_{N-1}\right\} \subset \mathbb{P}_{N}$ is no multisecant of $E$. Hence, denoting by $U \subset W$ the 2-dimensional subvector space defined by $P_{N-2} \subset \mathbb{P}\left(W^{*}\right)$, the evaluation map $U \otimes \mathcal{O}_{E} \rightarrow M^{n}\left(-\sum_{j=1}^{N-1} p_{j}\right)$ is surjective. Consider the following commutative diagram with exact rows and columns:

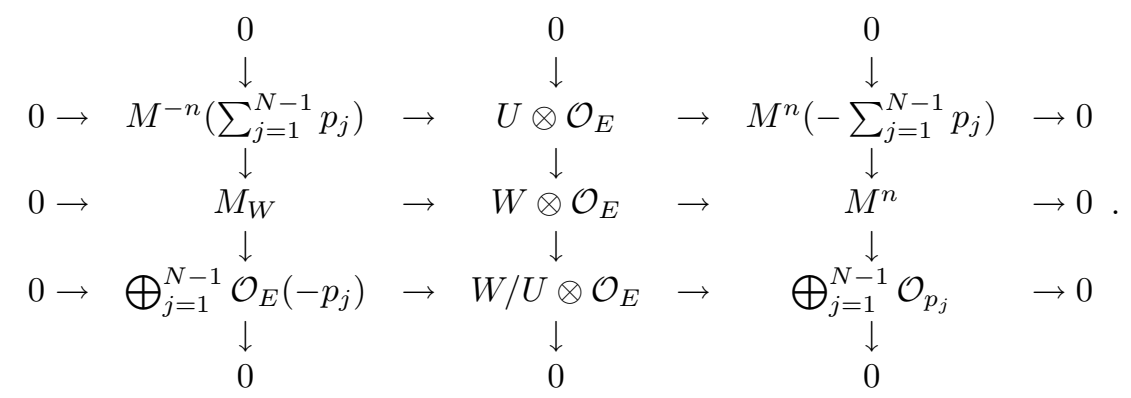

The left-hand vertical column yields the exact sequence

$$
0 \rightarrow M^{k-n}\left(\sum_{j=1}^{N-1} p_{j}\right) \otimes P \rightarrow M_{W} \otimes M^{k} \otimes P \rightarrow \bigoplus_{j=1}^{N-1} M^{k}\left(-p_{j}\right) \otimes P \rightarrow 0 .
$$

By assumption

$$
\operatorname{deg} M^{k-n}\left(\sum_{j=1}^{N-1} p_{j}\right) \otimes P=d(k-n)+N-1=d k-3>0
$$

and

$$
\operatorname{deg} M^{k}\left(-p_{j}\right) \otimes P=d k-1>0,
$$

and thus

$$
h^{1}\left(M^{k-n}\left(\sum_{j=1}^{N-1} p_{j}\right) \otimes P\right)=H^{1}\left(M^{k}\left(-p_{j}\right) \otimes P\right)=0 .
$$

This implies the assertion.

(6.5) Remark. The same proof yields

Suppose $(E, M)$ is a polarized elliptic curve and $W \subset H^{0}\left(E, M^{k}\right), n \geq 1, a$ $2-$ normal subvector space of codimension $c$. Then for all $P \in \operatorname{Pic}^{0}(E)$ and integers $k$ such that $k \operatorname{deg} M \geq c+3$ the canonical map

$$
W \otimes H^{0}\left(M^{k} \otimes P\right) \rightarrow H^{0}\left(M^{n+k} \otimes P\right)
$$

is surjective.

(6.6) Proposition. Let $n$ be an integer $\geq 3$ such that $n d_{g}$ is even $\geq 6$ and $c \leq n^{g-1}$. The general subvector space $V \subseteq H^{0}(L)$ of codimension c is $2-n o r m a l$, and for every integer $k \geq \max \left(2, \frac{4}{d_{g}}\right)$ and $P \in \operatorname{Pic}^{0}(X)$ the canonical map

$$
\mu_{P}: V \otimes H^{0}\left(M^{k} \otimes P\right) \rightarrow H^{0}\left(M^{n+k} \otimes P\right)
$$

is surjective. Equivalently,

$$
H^{i}\left(M_{V} \otimes M^{k} \otimes P\right)=0 \quad \text { for } \quad i \geq 1 .
$$


Proof. By semicontinuity it suffices to consider the case that $(X, M) \simeq\left(X_{1}, M_{1}\right) \times$ $\left(E, M_{2}\right)$ with $E$ an elliptic curve and $\operatorname{deg} M_{2}=d_{g}$ and a polarized abelian variety $\left(X_{1}, M_{1}\right)$ of type $\left(d_{1}, \ldots, d_{g-1}\right)$. Furthermore it suffices to establish the existence of a subvector space $V \subseteq H^{0}\left(M^{n}\right)$ of codimension $c \geq n^{g-1}$ satisfying the above stated properties.

Let $W \subset H^{0}\left(E, M_{2}^{n}\right)$ be a 1 -codimensional subvector space as in Lemma 6.4. Then

$$
V:=p_{X_{1}}^{*} H^{0}\left(X_{1}, M_{1}^{n}\right) \otimes p_{E}^{*} W \subset H^{0}\left(M^{k}\right)=H^{0}(L)
$$

is a subvector space of codimension $c:=h^{0}(L) / n d_{g} \geq n^{g-1}$.

$H^{0}\left(X_{1}, M_{1}^{n}\right)$ is $2-$ normal, since $M_{1}^{n}$ is normally generated (see [CAV], Theorem 7.3.1), and $W$ is $2-$ normal by assumption. The Künneth isomorphism implies that the following diagram is commutative:

$$
\begin{array}{ccc}
V \otimes V & \stackrel{\mu}{ } & H^{0}\left(L^{2}\right) \\
\downarrow \simeq & & \\
\downarrow \simeq & \longrightarrow \\
{\left[H^{0}\left(M_{1}^{n}\right) \otimes H^{0}\left(M_{1}^{n}\right)\right] \otimes[W \otimes W]} & \overrightarrow{\mu_{1} \otimes \mu_{2}} & H^{0}\left(M_{1}^{2 n}\right) \otimes H^{0}\left(M_{2}^{2 n}\right) .
\end{array}
$$

By what we have said above, $\mu_{1}$ and $\mu_{2}$ are surjective. Hence $V$ is $2-$ normal. Again by Künneth's theorem the following diagram commutes:

$$
\begin{array}{ccc}
V \otimes H^{0}\left(X, M^{k} \otimes P\right) & \stackrel{\mu_{p}}{\longrightarrow} & H^{0}\left(X, M^{n+k} \otimes P\right) \\
\downarrow \simeq & & \downarrow \simeq \\
{\left[H^{0}\left(X_{1}, M_{1}^{n}\right) \otimes H^{0}\left(X_{1}, M_{1}^{k} \otimes P_{1}\right)\right]} & & H^{0}\left(X_{1}, M_{1}^{n+k} \otimes P_{1}\right) \\
\otimes & \longrightarrow & \otimes^{0} \\
{\left[W \otimes H^{0}\left(E, M_{2}^{k} \otimes P_{2}\right)\right]} & & H^{0}\left(E, M_{2}^{n+k} \otimes P_{2}\right)
\end{array}
$$

for all $k$ and $P_{1} \in \operatorname{Pic}^{0}\left(X_{1}\right), P_{2} \in \operatorname{Pic}^{0}(E)$ such that $P=p_{X_{1}}^{*} P_{1} \otimes p_{E}^{*} P_{2}$. If $k \geq \frac{4}{d_{g}}$, then

$$
W \otimes H^{0}\left(E, M_{2}^{k} \otimes P_{2}\right) \rightarrow H^{0}\left(E, M_{2}^{n+k} \otimes P_{2}\right)
$$

is surjective according to Lemma 6.4. Moreover, [CAV], Proposition 7.3.4 implies the surjectivity of

$$
H^{0}\left(X_{1}, M_{1}^{n}\right) \otimes H^{0}\left(X_{1}, M_{1}^{k} \otimes P_{1}\right) \rightarrow H^{0}\left(X_{1}, M_{1}^{n+k} \otimes P_{1}\right)
$$

since $n \geq 3$ and $k \geq 2$. This shows that $\mu_{P}$ is surjective as well. Finally, the exact cohomology sequence of $0 \rightarrow M_{V} \otimes M^{k} \otimes P \rightarrow V \otimes M^{k} \otimes P \rightarrow M^{n+k} \otimes P \rightarrow 0$ and the fact that $H^{i}\left(M^{k} \otimes P\right)=H^{i}\left(M^{n+k} \otimes P\right)=0$ for $i \geq 1$ imply that the vanishings $H^{i}\left(M_{V} \otimes M^{k} \otimes P\right)=0$ for $i \geq 1$ are equivalent to the surjectivity of the maps $\mu_{P}$.

We need the following result due to Lazarsfeld (see [L2], Proposition 4.3.2):

(6.7) Lemma. Let $N$ be an ample line bundle and $E$ a vector bundle on $X$ satisfying

$$
H^{i}(E \otimes P)=H^{i}(E \otimes N \otimes P)=0
$$

for all $i \geq 1$ and $P \in \operatorname{Pic}^{0}(X)$. Then the canonical map

$$
\sum_{P \in P i c^{0}(X)} H^{0}(N \otimes P) \otimes H^{0}\left(E \otimes P^{-1}\right) \rightarrow H^{0}(E \otimes N)
$$

is surjective. 
Proof of Theorem 6.1 [respectively Remark 6.3]. The hypotheses of Proposition 6.6 are fulfilled; hence $V$ is 2 -normal and according to Theorem 2.4 it suffices to show that

$$
H^{1}\left(\bigwedge^{j} M_{V} \otimes L^{m}\right)=0
$$

for $1 \leq j \leq p+1$ and $m \geq 1$. Since the ground field is $\mathbb{C}$ the vector bundle $\bigwedge^{j} M_{V} \otimes L^{m}$ is a direct summand of $M_{V}^{\otimes j} \otimes L^{m}$ and thus $H^{1}\left(M_{V}^{\otimes j} \otimes L^{m}\right)=0$ for $1 \leq j \leq p+1$, and $m \geq 1$ implies (1). Here the following even stronger result will be proven.

Under the numerical conditions of Theorem 6.1

$$
H^{i}\left(M_{V}^{\otimes j} \otimes M^{m} \otimes P\right)=0
$$

for $i, j \geq 1, m \geq k_{0} j$ and all $P \in \operatorname{Pic}^{0}(X)$.

The proof of $(*)$ proceeds by induction on $j$. Suppose first $j=1$ : Since $n \geq 3$ and $m \geq k_{0}$, Proposition 6.6 implies $H^{i}\left(M_{V} \otimes M^{m} \otimes P\right)=0$ for $i \geq 1$, and $P \in \operatorname{Pic}^{0}(X)$. (This is just the hypothesis of Remark 6.3.)

Now suppose $(*)$ holds for some $j \geq 1$. Let $m \geq k_{0}(j+1)$ and $P \in \operatorname{Pic}^{0}(X)$. The exact cohomology sequence associated with $0 \rightarrow M_{V}^{\otimes(j+1)} \otimes M^{m} \otimes P \rightarrow V \otimes$ $M_{V}^{\otimes j} \otimes M^{m} \otimes P \rightarrow M_{V}^{\otimes j} \otimes M^{m+n} \otimes P \rightarrow 0$ implies together with the induction hypothesis that

$$
H^{i}\left(M_{V}^{\otimes(j+1)} \otimes M^{m} \otimes P\right)=0
$$

for $i \geq 2$ and that the following sequence is exact:

$$
\begin{aligned}
V \otimes H^{0}\left(M_{V}^{\otimes j} \otimes M^{m} \otimes P\right) & \stackrel{\mu}{\longrightarrow} H^{0}\left(M_{V}^{\otimes j} \otimes M^{m+n} \otimes P\right) \\
& \longrightarrow H^{1}\left(M_{V}^{\otimes}(j+1) \otimes M^{m} \otimes P\right) \rightarrow 0 .
\end{aligned}
$$

Hence it suffices to show that $\mu$ is surjective. For this consider the following commutative diagram:

$$
\begin{aligned}
& \sum_{Q \in P i c^{0}(X)}\left(\begin{array}{c}
V \otimes H^{0}\left(M^{k_{0}} \otimes P \otimes Q\right) \\
\otimes H^{0}\left(M_{V}^{\otimes j} \otimes M^{m-k_{0}} \otimes Q^{-1}\right)
\end{array}\right) \quad \longrightarrow \quad V \otimes H^{0}\left(M_{V}^{\otimes j} \otimes M^{m} \otimes P\right) \\
& \sum_{Q \in P i c^{0}(X)}\left(\begin{array}{c}
H^{0}\left(M^{n+k_{0}} \otimes P \otimes Q\right) \\
\otimes H^{0}\left(M_{V}^{\otimes j} \otimes M^{m-k_{0}} \otimes Q^{-1}\right)
\end{array}\right) \quad \stackrel{\mu_{2}}{\longrightarrow} \quad H^{0}\left(M_{V}^{\otimes j} \otimes M^{m+n} \otimes P\right) \text {. }
\end{aligned}
$$

The map $\mu_{1}$ is surjective according to Proposition 6.6. On the other hand, by the induction hypothesis

$$
H^{i}\left(M_{V}^{\otimes j} \otimes M^{m-k_{0}} \otimes P\right)=H^{i}\left(M_{V}^{\otimes j} \otimes M^{m+n} \otimes P\right)=0
$$

for all $P \in \operatorname{Pic}^{0}(X)$, since $m+n \geq m-k_{0} \geq k_{0}(j+1)-k_{0}=k_{0} j$. Hence $\mu_{2}$ (and thus $\mu$ as well) is surjective according to Lemma 6.7 (applied to $E=M_{V}^{\otimes j} \otimes M^{m-k_{0}}$ and $\left.N=M^{n+k_{0}} \otimes P\right)$. 


\section{Equations for Abelian Varieties in $\mathbb{P}_{N}$}

Let $(X, M)$ be a polarized abelian variety of type $\left(d_{1}, \ldots, d_{g}\right)$ such that $L=M^{n}$ is very ample for $n \geq 2$. The line bundle $L$ defines an embedding $\varphi_{L}: X \hookrightarrow \mathbb{P}_{N+1}=$ $\mathbb{P}\left(H^{0}(L)^{*}\right)$, the image of which is invariant with respect to the Heisenberg group associated with $L$. In [CAV], $\S \S 7.5$ and 7.6 , this action of the Heisenberg group is used to develop quadratic and cubic equations for $X$ in $\mathbb{P}_{N+1}$. The aim of this section is to apply these methods to noncomplete linear systems. For simplicity we consider here only the case of sublinear systems of codimension 1.

Let

$$
K(L)=K_{1} \oplus K_{2}
$$

be a decomposition into isotropic subgroups $K_{i} \simeq \mathbb{Z} / n d_{1} \mathbb{Z} \times \ldots \times \mathbb{Z} / n d_{g} \mathbb{Z}$. Let $P \in \mathbb{P}_{N+1}$ be a $K_{2}$-invariant point. The linear projection

$$
p r: \varphi(X) \rightarrow \bar{X} \subset \mathbb{P}_{N}
$$

with center $P$ is $K_{2}$-equivariant. We will develop quadratic and cubic equations for $\bar{X}$. Moreover we will discuss when these equations generate the homogeneous ideal of $\bar{X}$ in $\mathbb{P}_{N}$.

Let $\mathcal{G}_{2} \subset \mathcal{G}(L)$ be a section of the theta group of $L$ over $K_{2}$ and $H^{0}(L)=$ $\bigoplus_{\chi \in \mathcal{G}_{2}^{*}} U_{\chi}$ the decomposition into eigenspaces (as in Section 4). The projection pr defines a subvector space $V \subset H^{0}(L)$ of codimension 1 such that the following diagram commutes:

$$
\begin{array}{rcrl}
X & \stackrel{\varphi_{L}}{\longrightarrow} & \varphi_{L}(X) & \subset \mathbb{P}_{N+1} \\
\varphi \searrow & \downarrow \text { pr } & \\
& \bar{X} & \subset \mathbb{P}\left(V^{*}\right)=\mathbb{P}_{N}
\end{array}
$$

In particular, $V$ is invariant with respect to the action of the group $\mathcal{G}_{2}$. Hence (eventually translating the line bundle $L$ ) we may assume that $L$ is of characteristic 0 with respect to (1) and

$$
V=\bigoplus_{\chi \in \mathcal{G}_{2}^{*}-\{0\}} U_{\chi}
$$

Quadratic and cubic equations for $\bar{X}$ are the elements of the vector spaces $I(V)_{i}=$ $\operatorname{ker}\left\{S^{i} V \rightarrow H^{0}\left(L^{i}\right)\right\}, i=2,3$. Obviously $I(V)_{i}$ is a subvector space of $I(L)_{i}=$ $\operatorname{ker}\left\{S^{i} H^{0}(L) \rightarrow H^{0}\left(L^{i}\right)\right\}$, and by elimination theory $I(V)_{i}$ is the intersection of $I(L)_{i}$ with $S^{i} V \subset S^{i} H^{0}(L)$. The generators of $I(L)_{2}$ and $I(L)_{3}$ are the classical Riemann Theta relations and the cubic Theta relations, respectively (see [CAV], Sections 7.5 and 7.6 ).

We need some further notation: For $\nu=2,3$ and 6 let $K\left(L^{\nu}\right)=K\left(L^{\nu}\right)_{1} \oplus K\left(L^{\nu}\right)_{2}$ be decompositions into isotropic subgroups compatible with (1), i.e. $\nu K\left(L^{\nu}\right)_{i}=K_{i}$ for $i=1,2$. Let $\left\{\vartheta_{x}^{L^{\nu}} \mid x \in K\left(L^{\nu}\right)_{1}\right\} \subset H^{0}\left(L^{\nu}\right)$ be bases of canonical theta functions in the sense of [CAV], Theorem 3.2.7, and denote

$$
\begin{aligned}
& \theta_{y, \rho}:=\sum_{z \in K_{1} \cap X_{2}} \rho(z) \vartheta_{y+z}^{L^{2}} \in H^{0}\left(L^{2}\right) \\
& \theta_{\left(y_{1}, y_{2}\right), \sigma}:=\sum_{z \in K\left(L^{6}\right)_{1} \cap X_{6}} \sigma(z) \vartheta_{y_{1}-z}^{L^{6}} \otimes \vartheta_{y_{1}-3 z}^{L^{2}} \in H^{0}\left(L^{6}\right) \otimes H^{0}\left(L^{2}\right)
\end{aligned}
$$


with elements $y, y_{2} \in K\left(L^{2}\right)_{1}, y_{1} \in K\left(L^{6}\right)_{1}, \rho \in \operatorname{Hom}\left(K_{1} \cap X_{2}, \mathbb{C}^{*}\right)$, and $\sigma \in$ $\operatorname{Hom}\left(K\left(L^{6}\right)_{1} \cap X_{6}, \mathbb{C}^{*}\right)$. (Here $\left.X_{n}=\{x \in X \mid n x=0\}\right)$. For $x \in K_{1}$ denote by $X_{x}$ the coordinate function of $\mathbb{P}\left(H^{0}(L)^{*}\right)=\mathbb{P}_{N+1}$ (respectively of $\mathbb{P}\left(V^{*}\right)$ if $x \neq 0$ ) corresponding to the basis element $\vartheta_{x}^{L} \in H^{0}(L)$. With this notation pr: $\mathbb{P}_{N+1}--\rightarrow \mathbb{P}_{N}$ is the projection defined by eliminating $X_{0}$.

(7.1) Proposition. a) Suppose $L=M^{n}$, and $n \geq 4$ is even. The quadratic equations

$$
\begin{aligned}
& \theta_{y_{1}, \rho}(0) \sum_{z \in K_{1} \cap X_{2}} \rho(z) X_{y+y_{2}+z} X_{y-y_{2}+z} \\
& =\theta_{y_{2}, \rho}(0) \sum_{z \in K_{1} \cap X_{2}} \rho(z) X_{y+y_{1}+z} X_{y-y_{1}+z}
\end{aligned}
$$

for $y, y_{1}, y_{2} \in K\left(L^{2}\right)_{1}$ satisfying $y \pm y_{i} \in K_{1}-\left(K_{1} \cap X_{2}\right)$ and $\rho \in \operatorname{Hom}\left(K_{1} \cap X_{2}, \mathbb{C}^{*}\right)$ generate the vector space $I(V)_{2}$.

b) Suppose $L=M^{3 k}, k \geq 1$. The cubic equations

$$
\begin{aligned}
& \theta_{\left(y_{1}, y_{2}\right), \sigma}(0) \sum_{z \in K\left(L^{6}\right)_{1} \cap X_{6}} \sigma(z) X_{y_{1}^{\prime}+y_{2}^{\prime}+y_{3}+2 z} X_{y_{1}^{\prime}-y_{2}^{\prime}+y_{3}+2 z} X_{-2 y_{1}^{\prime}+y_{3}+2 z} \\
& =\theta_{\left(y_{1}^{\prime}, y_{2}^{\prime}\right), \sigma}(0) \sum_{z \in K\left(L^{6}\right)_{1} \cap X_{6}} \sigma(z) X_{y_{1}+y_{2}+y_{3}+2 z} X_{y_{1}-y_{2}+y_{3}+2 z} X_{-2 y_{1}+y_{3}+2 z}
\end{aligned}
$$

for $y_{1}, y_{1}^{\prime} \in K\left(L^{6}\right)_{1}, y_{2}, y_{2}^{\prime} \in K\left(L^{2}\right)_{1}, y_{3} \in K\left(L^{3}\right)_{1}$ satisfying $y_{1} \pm y_{2}+y_{3}, y_{1}^{\prime} \pm y_{2}^{\prime}+$ $y_{3}, 2 y_{1}-y_{3}, 2 y_{1}^{\prime}-y_{3} \in K_{1}-\left(K_{1} \cap X_{3}\right)$ and $\sigma \in \operatorname{Hom}\left(K\left(L^{6}\right)_{1} \cap X_{6}, \mathbb{C}^{*}\right)$ generate the vector space $I(V)_{3}$.

Proof. The above equations are exactly those Riemann (respectively cubic) equations, not containing the variable $X_{0}$.

Finally we investigate when the above equations generate the ideal $I(V)$ of $\bar{X}$ in $\mathbb{P}\left(V^{*}\right)$. With the notation as above we have

(7.2) Proposition. Suppose $(X, L)$ is a general polarized abelian variety. If $d_{g} \geq 2$ and $n \geq 4$ is even, then $V=\bigoplus_{\chi \neq 0} U_{\chi} \subset H^{0}(L)$ satisfies property $\widetilde{N}_{1}$, i.e. the ideal $I(V)$ is generated by the quadratic equations 9.1 (a).

Proof. According to Remark 6.3 it suffices to show that

a) $V$ is 2 -normal, and

b) $H^{i}\left(M_{V} \otimes M^{k} \otimes P\right)=0$

for $i \geq 1, k \geq 2$ and $P \in \operatorname{Pic}^{0}(X)$.

By semicontinuity it suffices to consider the case $(X, M)=\left(X_{1}, M_{1}\right) \times\left(E, M_{2}\right)$ with a polarized abelian variety $\left(X_{1}, M_{1}\right)$ of type $\left(d_{1}, \ldots, d_{g-1}\right)$ and an elliptic curve $E$ with a line bundle $M_{2}$ of degree $d_{g}$. Choose a decomposition of $K\left(M_{2}^{n}\right) \subset E$ compatible with (1),

$$
K\left(M_{2}^{n}\right)=A_{1} \oplus A_{2},
$$

i.e. $A_{i} \subseteq K_{i}$ and $A_{i} \simeq \mathbb{Z} / n d_{g} \mathbb{Z}$ for $i=1,2$. Consider the corresponding decomposition of $H^{0}\left(E, M_{2}^{n}\right)$ into eigenspaces

$$
H^{0}\left(E, M_{2}^{n}\right)=\bigoplus W_{\chi}
$$


Here the sum runs over all characters $\chi$ of a section $\tilde{A}_{2} \subset \mathcal{G}\left(E, M_{2}^{n}\right)$ over $A_{2}$. The subvector space

$$
W:=\bigoplus_{\chi \neq 0} W_{\chi} \subset H^{0}\left(E, M_{2}^{n}\right)
$$

is of codimension 1 and is $2-$ normal according to Theorem 4.1. By Remark 6.5 the canonical map

$$
W \otimes H^{0}\left(E, M_{2}^{k} \otimes P_{2}\right) \rightarrow H^{0}\left(M^{n+k} \otimes P_{2}\right)
$$

is surjective for $k \geq 2$ and $P_{2} \in \operatorname{Pic}^{0}(E)$. On the other hand,

$$
H^{0}\left(X_{1}, M_{1}^{n}\right) \otimes H^{0}\left(X_{1}, M_{1}^{k} \otimes P_{1}\right) \rightarrow H^{0}\left(X_{1}, M_{1}^{n+k} \otimes P_{1}\right)
$$

is surjective for $k \geq 2$ (and even for $n \geq 3$ ), and $P_{1} \in \mathrm{Pic}^{0}\left(X_{1}\right)$ by [CAV], Proposition 7.3.4. For $k=n$ and $P_{1}=0$ this implies that

$$
\left(p_{X_{1}}^{*} H^{0}\left(X_{1}, M_{1}^{n}\right) \otimes p_{E}^{*} W\right)^{\otimes 2} \rightarrow p_{X_{1}}^{*} H^{0}\left(X_{1}, M_{1}^{2 n}\right) \otimes p_{E}^{*} H^{0}\left(E, M_{2}^{2 n}\right) \simeq H^{0}\left(X<L^{2}\right)
$$

is surjective. Since $p_{X_{1}}^{*} H^{0}\left(X_{1}, M_{1}^{n}\right) \otimes p_{E}^{*} W \subset V$, the vector space $V$ is $2-$ normal. Similarly, Künneth's isomorphism yields the surjectivity of

$$
V \otimes H^{0}\left(X_{1} \times E, M^{k} \otimes P\right) \rightarrow H^{0}\left(X_{1} \times E, M^{n+k} \otimes P\right)
$$

for $k \geq 2$ and $P \in \operatorname{Pic}^{0}\left(X_{1} \times E\right)$. Now the exact cohomology sequence of

$$
0 \rightarrow M_{V} \otimes M^{k} \otimes P \rightarrow V \otimes M^{k} \otimes P \rightarrow M^{n+k} \otimes P \rightarrow 0
$$

implies b).

If $X$ is an abelian surface, i.e. $g=2$, this gives

(7.3) Proposition. Suppose $n \geq 3$ and $n d_{2} \geq 6$ is even. Then the ideal $I(V)$ is generated by quadrics and cubics.

Proof. As in the proof above, one sees that $V=\bigoplus_{\chi \neq 0} U_{\chi}$ is $2-$ normal. Since

$$
h^{0}(L)=n^{2} d_{1} d_{2} \geq 18 \geq 3+9,
$$

$V$ satisfies $\widetilde{N}_{0}$ by Theorem 5.1, and Theorem 5.3 yields the assertion.

\section{REFERENCES}

[ACGH] E. Arbarello, M. Cornalba, P.A. Griffiths, J. Harris: Geometry of Algebraic Curves I, Grundlehren 267, Springer Verlag (1985) MR 86h:14019

[B] Ch. Birkenhake: Linear Systems on Projective spaces, Manuscripta Math. 88 (1995) 177-184.

[CAV] H. Lange, Ch. Birkenhake: Complex Abelian Varieties, Grundlehren 302, Springer Verlag (1992) MR 94j:14001

[G1] M Green: Koszul cohomology and the geometry of projective varieties, J. Diff. Geom. 19 (1984) 125 - 171 MR 85e: 14022

[G2] M. Green: Koszul cohomology and Geometry, in: Lectures on Riemann surfaces, Proceedings of a conference in Trieste, World Scientific 1989, 177-200 MR 91k:14012

[G-L] M. Green, R. Lazarsfeld: Some results on the syzygies of finite sets and algebraic curves, preprint

[Ha] R. Hartshorne: Algebraic Geometry, Springer Verlag (1977) MR 57:3116

[Hi] F. Hirzebruch: Topological Methods in Algebraic Geometry, Grundlehren 131, Springer Verlag (3rd ed., 1966) MR 34:2573

[K] G. Kempf: The projective coordinate ring of abelian varieties, in: Algebraic Analysis, Geometry and Number Theory (ed. by J. I. Igusa), The Johns Hopkins Press (1989), 225 $-236$ 
[L1] R. Lazarsfeld: A Sampling of Vector Bundle Techniques in the Study of Linear Series, in: Lectures on Riemann Surfaces, Proceedings of a conference in Trieste, World Scientific 1989, $550-559$ MR 92f: 14006

[L2] R. Lazarsfeld: Syzygies of Abelian Varieties, private notes (1993)

[Ma] H. Matsumura: Commutative Algebra, Benjamin/Cummings Publishing Company (1980) MR 82i:13003

[M] D. Mumford: Varieties defined by quadratic equations, in: Questions on Algebraic varieties, C.I.M.E. (1970) 29 - 100 MR 44:209

Mathematisches Institut, Universität Erlangen Bismarckstrasse $1 \frac{1}{2}$, D-91054 ErlanGen, Germany

E-mail address: Birkenhake@mi.uni-erlangen.de 